\title{
Computational Screening of Chiral Organic Semiconductors: Exploring Side-Group Functionalization and Assembly to Optimize Charge Transport
}

Julia A. Schmidt, Joseph A. Weatherby, Isaac J. Sugden, Alejandro Santana-Bonilla, Francesco Salerno, Matthew J. Fuchter, Erin R. Johnson, Jenny Nelson, and Kim E. Jelfs*

Cite This: Cryst. Growth Des. 2021, 21, 5036-5049

Read Online

ACCESS | Lلll Metrics \& More | 回 Article Recommendations | st Supporting Information

ABSTRACT: Molecular materials are challenging to design as their packing arrangements, and hence their properties, are subject to subtle variations in the interplay of soft intermolecular interactions. Rational design of new molecular materials with tailored properties is currently hampered by the difficulty in predicting how a candidate molecule will pack in space and how to control the particular polymorph obtained experimentally. Here, we develop a rapid screening approach to aid the material design process, which is then applied to predict the charge-transfer properties of 1344 helicene compounds that have potential as

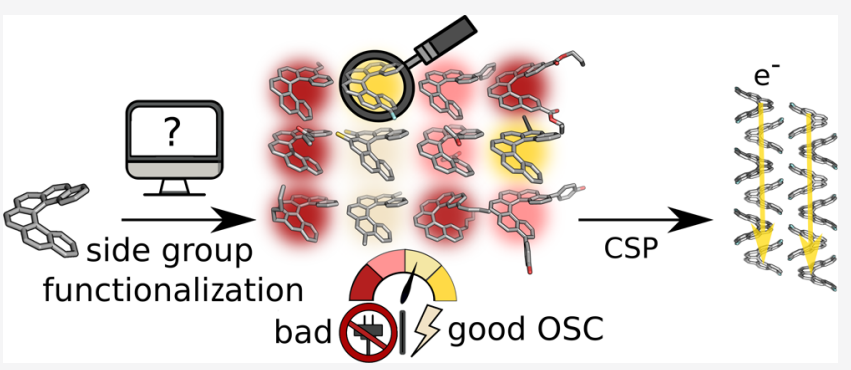
organic electronic materials. Our approach bridges the gap between single-molecule design, molecular assembly, and the resulting charge-carrier mobilities. We find that fluorination significantly improves electron transport in the molecular material by over $200 \%$, while side groups containing triple bonds largely lead to improved transfer integrals. We validate our screening approach through the use of full crystal structure prediction for the most promising compounds to confirm the presence of favorable packing motifs that maximize charge mobility.

\section{INTRODUCTION}

Organic molecular materials hold promise as low-cost organic semiconductors (OSCs) due to their favorable magnetic, electrical, and optical properties. Development of OSCs offers technologies with new functionality, such as flexible ultrathin displays, biodegradable electronics, and energy-harvesting smart materials. OSC performance depends on the ease with which charge carriers can move across the $\pi$-stacked systems of the component molecules, which is predominantly influenced by how they are arranged within the solid material, ${ }^{1,2}$ as well as the energies of their frontier orbitals. The soft intermolecular interactions in molecular materials typically give rise to many isolable solid forms, or polymorphs, within a small energy window, the structures of which are difficult to predict from first principles. Changes in molecular packing between different polymorphs can result in large variations in physical properties.

Materials scientists are continually searching for organic semiconducting molecules with solid forms that give rise to high charge-carrier mobilities and good processability., ${ }^{2,3}$ Previous methods to screen small molecules for potential suitability as OSCs include data mining, ${ }^{4,5}$ atomistic substitutions, ${ }^{6}$ and machine-learning approaches. ${ }^{7,8}$ For example, Kunkel et al. screened the Cambridge Structural Database (CSD) to obtain statistically significant structureproperty relationships, with certain scaffolds (side groups) leading to consistently improved charge-transport properties. ${ }^{4}$ Also, atomistic substitution leading to a modified polymorph landscape, and thus changed charge-transport behavior, has been experimentally investigated by Sorli et al. ${ }^{6}$ From a computational perspective, Pulido et al. combined crystal structure and property prediction to build energy-structurefunction maps that describe the possible polymorphs of a candidate molecule and their respective properties. 90

Recently, crystal structure prediction (CSP) has found increasing use in the materials community. ${ }^{11-14}$ Firstprinciples CSP refers to the computational prediction of the likely crystal structures of a molecule, starting from nothing more than the atom connectivity. The most common strategy is a multidimensional search of the crystal-energy landscape for the local minima corresponding to different crystal structures. ${ }^{15,16}$ All tentative crystal structures are then ranked by their lattice ${ }^{17}$ or free energy to determine the thermodynamically most stable structures using classical force fields. ${ }^{18}$ The

Received: April 23, 2021

Revised: May 24, 2021

Published: August 8, 2021 


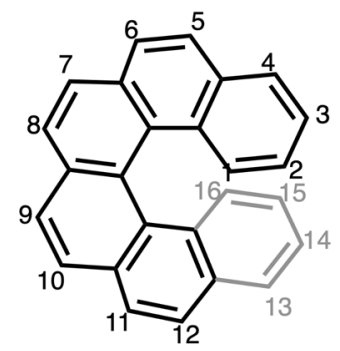

a) $M$-[6]helicene

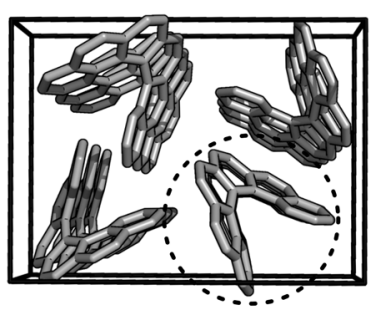

b) experimental crystal structure (HEXHEL)

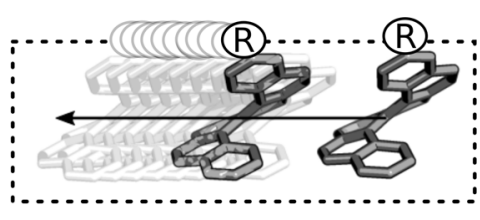

(R) = halogens, alcohols, ethers, esters, alkyl groups, etc.

c) translational dimer

Figure 1. (a) Structure of the $M$-[6]helicene backbone, which is mono- and symmetrically disubstituted with different functional groups in all positions (1-16) during screening. (b) Experimental crystal structure of [6] helicene (front view), with the circled translational motif in the plane of the page. (c) Translational motif of the functionalized [6] helicene (side view) for the particular case of monosubstitution in the 3-position.

lowest-energy crystal structures can be reranked more accurately, for example, using periodic dispersion-corrected density functional theory (DFT-D) calculations at $0 \mathrm{~K}^{19,20}$ Finally, thermal free-energy corrections can be applied to the lowest-energy candidates. ${ }^{21,22}$ The relationship between molecular structure, crystal packing, low-frequency vibrations, and charge transport still poses many open questions, and there would be a significant value in developing a framework to design these materials. ${ }^{23,24}$

We are particularly interested in chiral helicene molecules as potential OSCs. Helicenes are axially fused benzene rings, as shown for [6]helicene in Figure 1a. Due to the chirality present, a [6] helicene molecular crystal can either contain only one chiral form (enantiopure) or molecules of both handedness (racemic). Small-molecule chirality can be used in OSCs to provide added opportunities from chiral compositiondependent changes to bulk properties, ${ }^{25}$ chirality-driven crystallization of racemic over enantiopure candidates, ${ }^{26}$ and the control of spin for use in chiral-induced spin selectivity filters. ${ }^{27}$ Helicenes have already been successfully tested for applications in chiroptical switches, ${ }^{28}$ circularly polarized (CP) OLEDs, ${ }^{29} \mathrm{CP}$ light photodetectors, ${ }^{30}$ and transistor materials. $^{31}$ This suggests future usage of helicenes in a large range of potential applications. For example, in OLED screen development, chiral materials have generated interest for use in the preparation of CP-OLEDs. CP-OLEDs provide a means to address energy losses by commonly used antiglare filters, which eliminate glare from external light sources (e.g., sunlight). ${ }^{25}$

Previously, we performed CSP studies to predict the carbo[6]helicene and aza[6] helicene crystal-energy landscapes and found that the chirality of the crystal (enantiopure vs racemic) can drastically affect the corresponding charge-carrier mobilities. ${ }^{31,32}$ For carbo[6] helicene, we structurally classified polymorphs into recurring packing motifs. A particular "translational motif" (Figure 1c) was found with the highest probability across the entire crystal-energy landscape, being observed in $63 \%$ of the hypothetical low-energy polymorphs. ${ }^{32}$ Most recently, Salerno et al. studied the effect of the nitrogen substitution position on charge transport for the most commonly observed [6] helicene crystal structure (CSD code: HEXHEL, Figure $1 \mathrm{~b})^{33}$ and found that this same "translational motif" gave rise to the highest electron-transfer integrals across the majority of aza[6] helicene isomers.

While one may design a material that has promising properties, unless the thermodynamic viability and thus the synthetic route to the material has been considered, it is unlikely that the material will actually be obtained. ${ }^{34}$ Instead, one must screen thousands of potential materials, considering viability and then their properties. ${ }^{35}$ Thus, in the case of organic semiconductors, if we wish to "design" a molecule with a target property, we must consider the solid-state packing. In practical terms, to include the effect of packing within a highthroughput computational study, as we attempt to do here, it is essential to make some approximations and consider a subset of possible structural degrees of freedom.

In this work, we leverage the ubiquity of the translational motif for helicene molecules to screen for high charge mobility across $\sim 1300$ molecules. We focus on investigating the helicenes as n-type semiconductors, examining their electron mobilities, because [6] helicenes tend to be better electrontransporting than hole-transporting materials based on our computational predictions. ${ }^{32}$ Since CSP is too computationally exhaustive to screen for large numbers of new molecular materials, the computed transport across a translated-dimer model serves as a proxy to predict charge transport in the solid material. Computational screening via the simplified dimer model, based on one of the most common [6] helicene dimer motifs, elucidates (i) a molecule's tendency to adopt the chosen translational motif, (ii) its suitability to maximize charge mobility, (iii) effects of substitution position for a given functional group, and (iv) whether asymmetric or symmetric disubstitution is preferable. The translational dimer motif is chosen for screening both because of its relative abundance and because translated dimers often yield the largest transfer integral at a given molecular separation. For the most promising compounds, we perform a full CSP search and compute the electron mobilities for the lowest-energy polymorphs, validating our approach. The overarching goal of this research is to uncover structure-property relationships to aid the design of new electronic materials with tailored properties.

\section{EXPERIMENTAL SECTION}

2.1. Compound Screening. 2.1.1. Functional-Group Selection and Dimer Generation. The M-[6] helicene backbone was systematically monosubstituted in all 16 possible hydrogen positions (1-16, as shown in Figure 1a) and disubstituted in all 8 possible symmetric configurations (1,16-, 2,15-, 3,14-, 4,13-, 5,12-, 6,11-, 7,10-, and 8,9disubstituted), to study the effect of functionalization on electron transport. We selected a range of common functional groups, such as halogens, alcohols, esters, ethers, carboxylic acids, phenyl groups, and alkyl groups, with the full set of substituents shown in Figures S3 and S4. We focused on side groups of small to medium size compared to the size of the [6]helicene backbone, such that, while new intermolecular interactions introduced by the side group may influence to molecular assembly, ${ }^{36}$ the interactions involving the [6]helicene backbone are still the main factors determining the crystal 
packing. The number of substitution patterns (24) and functional groups $(56)$ resulted in a total of $1344(24 \times 56)$ compounds studied.

To screen 1344 synthetically unknown [6]helicenes for their suitability as OSC materials, we first assume that the electronic coupling observed across one single translational dimer is sufficient to qualitatively assess the relative transport properties of the candidate molecular materials. The translational motif (Figure 1c) in [6]helicenes was first identified by Rice et al. and is more common than other dimer motifs, such as the interlocked dimer, the herringbone dimer, or the homo and heterochiral back-to-back dimer (Figure S1). ${ }^{32}$ The translational dimer is, as the name suggests, a purely homochiral ( $M$ - or $P$-handedness only) motif, where one molecule is translated with respect to the other along a vector perpendicular to the plane of the helix, and is characterized by good $\pi-\pi$ overlap of the adjacent [6] helicene backbones (Figure 1c). This translational motif was chosen due to its common occurrence in previously studied [6]helicene polymorphs ${ }^{31-33}$ and across [6] helicene molecules deposited in the CSD (see Section S1.2, Table S1 and Figure S2) - indeed, it is the most commonly occurring one-dimensional motif in these systems. It is, therefore, an informed guess that a new hypothetical [6] helicene might contain the translational packing motif in low-energy, synthetically achievable, polymorphs. We test this assumption later in our work by performing full CSP searches to determine the packing motifs observed in the polymorphic landscapes of the most promising candidate molecules. The choice of the translational dimer is further supported by a previous work on TIPSPn thin films in which the strongest vibrational mode corresponds to the translational vibration of the pentacene backbone along the main stacking axis (a translational motif). ${ }^{37}$ We note that the translational dimer motif will generally occur in any molecular crystal.

The generalized screening procedure is illustrated in Figure 2. A single $M$-carbo[6] helicene molecule was extracted from the

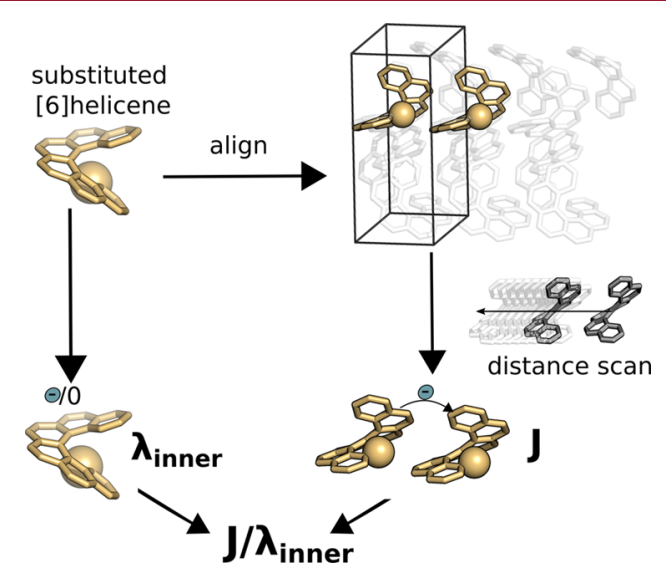

Figure 2. Overview of the screening procedure. A side group of choice is attached to the helicene backbone (top left) with the molecular geometry optimized. The substituted [6] helicene is then mapped back onto the unit cell, with the translational dimer shown along the $c$-axis, in the plane of the page. An intermolecular distance scan for this dimer is performed by modifying the $c$-axis length. The transfer integral $(J)$ is computed at the minimum-energy distance for the dimer, while the inner reorganization energy $\left(\lambda_{\text {inner }}\right)$ is computed from only the optimized single-molecule geometries for the neutral and anionic states. The ratio of these quantities is calculated as one measure of OSC suitability.

experimental carbo[6]helicene crystal structure (CSD code: HEXHEL, Figure $1 \mathrm{~b}$ ) and either monosubstituted or symmetrically disubstituted with a new side group. A short molecular dynamics (MD) simulation with the OPLS3e force field ${ }^{38}$ was performed to obtain a realistic starting geometry for DFT optimization, which is important in the case of flexible side groups that can adopt multiple conformers. The full MD details and sampling information can be found in Section S2.1 The geometry of the substituted [6]helicene molecule was then optimized at the $\mathrm{B} \mathrm{LYP}^{39} / 6-31 \mathrm{G}(\mathrm{d})$ level of theory using Gaussian $16 .^{40}$ The optimized molecular geometry was superimposed back onto the original [6] helicene position inside the experimentally observed HEXHEL crystal structure, using a python open-source toolkit for cheminformatics (RDKit). ${ }^{41}$

Translational dimers, based on those observed in the experimental carbo[6]helicene crystal structure, were extracted for each of the substituted [6]helicene molecules. As the intermolecular distance for the unsubstituted [6] helicene is not necessarily appropriate for the functionalized molecules, we performed a one-dimensional intermolecular distance scan along the $c$-axis of the crystal (Figure 3) from

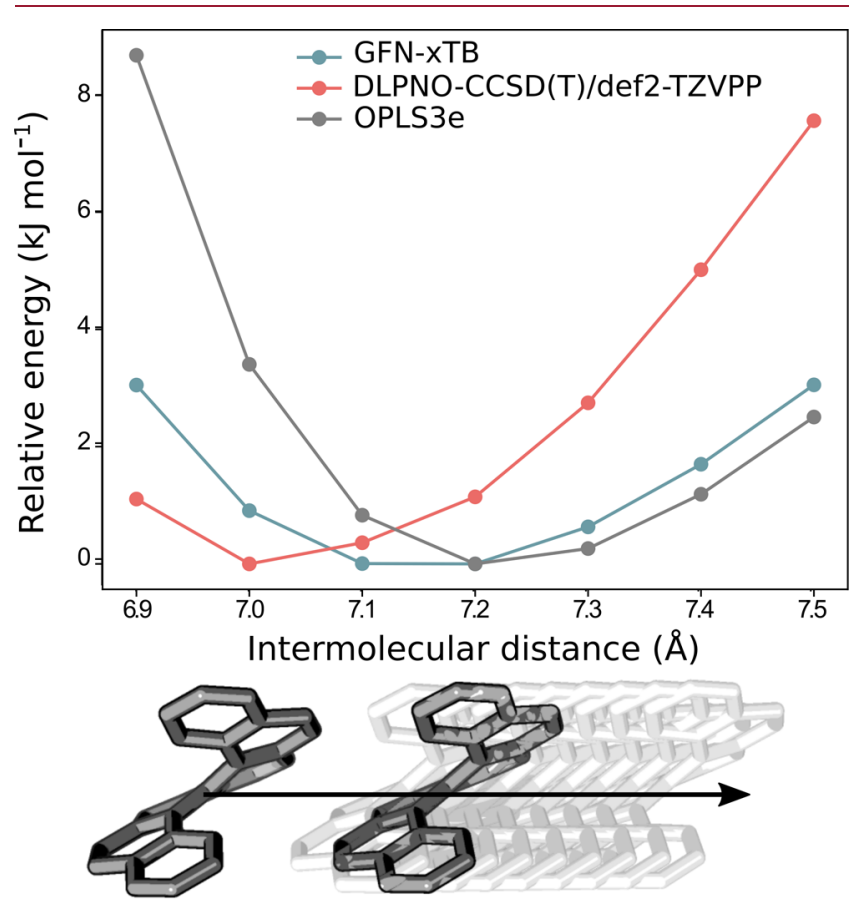

Figure 3. Intermolecular distance scans for the carbo[6]helicene translational dimer at three different levels of theory: the DLPNO$\operatorname{CCSD}(\mathrm{T})$ reference, the GFN-xTB semi-empirical method, and the OPLS3e force field. A rigid scan along the $c$-axis of the HEXHEL crystal structure (as shown by the arrow) was performed, with the lowest-energy dimer highlighted in dark gray.

6 to $14 \AA$, in $0.1 \AA$ increments, for each compound. The resulting minimum-energy distance was then used in all further property computations. The semi-empirical tight-binding method GFN-xTB ${ }^{42}$ was chosen as a cheap, but powerful, compromise to perform the distance scans. High-level DLPNO-CCSD $(\mathrm{T}),{ }^{43}$ or even conventional DFT with large basis sets and a Grimme-D3 dispersion correction, ${ }^{44}$ is too computationally costly to apply to 1344 molecules. Thus, we benchmarked the accuracy of the semi-empirical GFN-xTB and the OPLS3e force field, compared to DLPNO-CCSD $(T),{ }^{43}$ for the potential energy scan of carbo[6]helicene (Figure 3) and two terminally substituted [6] helicenes (Figures S7 and S8). We found that the minimum-energy distances, $d_{\text {min }}$ at the DLPNO-CCSD $(\mathrm{T})$ and GFN-xTB levels of theory consistently varied only by around 0.1 $\AA$, validating the choice of GFN-xTB.

2.1.2. Property Prediction. For each molecular dimer at its minimum-energy separation, we computed the transfer integrals by projecting the computed orbitals of the dimer onto the unperturbed localized orbitals of the individual molecules. ${ }^{32,45}$ The transfer integral is a measure of the electron (or hole) coupling between two adjacent interacting molecules

$$
J_{\text {elec }}=\langle i|\hat{\mathbf{F}}| j\rangle
$$

where $\hat{\mathbf{F}}$ denotes the Fock matrix of the dimer system, while $|i\rangle$ and $|j\rangle$ represent the orbitals localized on the molecules $i$ and $j$, respectively. 
All transfer integrals were computed with B3LYP/6-31G(d) using Gaussian $16{ }^{40}$ This level of theory has been proven successful in adequately describing the transfer integrals for [6]helicenes ${ }^{32}$ and other organic semiconductors. ${ }^{45}$ For full details of the transfer integrals and how they are employed using non-adiabatic, semiclassical Marcus theory, see Section S3.4.

A concern with using the translational dimer model as a proxy to screen for high bulk charge mobility is the distance dependence of the computed properties. It is commonly understood that transfer integrals exponentially decrease with the intermolecular distance, and this is seen in this work as well for the three test cases of [6]helicene, 2,15-dicyano[6]helicene, and 2,15-diethynyl[6]helicene (Figures S6, S7b, and S8b). This raises the question of whether the transfer integrals should be normalized by the distance, to enable comparison across compounds and deconvolute the impact of substitution from the distance dependence. However, the optimized dimer separations are our best approximation to the realistic intermolecular distances found in the bulk materials, and these will necessarily vary with molecular size and charge distribution, depending on the side groups. Thus, we do not normalize the transfer integrals and only compare results at each dimer's minimumenergy separation.

In addition to computing the transfer integrals, the inner electronic reorganization energy $\lambda_{\text {inner }}^{\text {elec }}$ was computed for each individual molecule using the four-point-method ${ }^{46}$

$$
\lambda_{\text {inner }}^{\text {elec }}=E_{\mathrm{n}}^{-}-E_{\mathrm{c}}^{-}+E_{\mathrm{n}}^{0}-E_{\mathrm{c}}^{0}
$$

Here, single-point energies were computed for the anionic or neutral states ( - or 0 , as indicated by the superscripts) at either the charged or neutral optimized geometries ( $c$ or $n$, as denoted by the subscripts). The reorganization energy is a quantitative measure of the ability of a single molecule to accommodate a negative charge, and the energetic penalty for conversion between the charged to the neutral geometries.

For a total of 56 of the 1344 molecules (4\%), at least one of the single-point energy calculations required to compute the inner reorganization energy did not converge. These examples were excluded from the subsequent analysis. However, to make sure that we do not miss any promising molecules, we used machine-learning regression methods to predict the missing inner reorganization energies based on our existing data. We compared both regression using XGBoost ${ }^{47}$ and kernel ridge regression techniques ${ }^{48}$ on Morgan radial fingerprints ${ }^{49}$ and managed to describe up to $90 \%$ of the variance using the latter (see Section S2.4).

There are several ways that the OSC suitability of a candidate molecule can be assessed based on the properties we have computed. Some approaches focus more on variance of $J$ rather than the inner reorganization energy, or vice versa. ${ }^{4}$ To combine the intra- and intermolecular aspects of charge transport in a single parameter, we choose the ratio $J_{\text {elec }} / \lambda_{\text {inner }}^{\text {elec }}$ to quantify the relative trends and identify promising candidate molecules. The higher the ratio, the higher the suitability of the molecule as an electron-transporting OSC. The same methodology could also be applied to hole transport, but for the purpose of this study, we focus on electron transport only.

As an alternative screening metric, we also consider the electron mobilities, $\mu$, obtained from non-adiabatic Marcus theory in the lowfield limit. While a number of theoretical models for describing charge transport in different regimes exist, Marcus theory ${ }^{50,51}$ is one of the most popular methods to describe charge transport in OSCs. ${ }^{24}$ It expresses charge hopping between weakly coupled sites with localized charges (hopping regime, $\left|J_{i j}\right| \ll \lambda$ ) and complements band transport theory, which instead assumes delocalized charges in the lowtemperature limit (band regime). ${ }^{24}$ Assuming a periodic onedimensional array of helicenes, regularly spaced along the axis that defines the translational dimer, the charge-carrier mobility along the chain can be calculated as an approximation to the mobility in the bulk material. In the low-field limit, the mobilities can be obtained from the transfer integral $(J)$, the reorganization energy $(\lambda)$, and the intermolecular center-of-mass distance $(a)$, as

$$
\mu=\frac{|J|^{2}}{\hbar} \sqrt{\frac{\pi}{\lambda k_{\mathrm{B}} T}}\left(\frac{e a^{2}}{k_{\mathrm{B}} T}\right) \exp \left(-\frac{\lambda}{4 k_{\mathrm{B}} T}\right)
$$

with more detail given in Section S2.5.

The absolute value of the mobility depends on the reorganization energy, one component of which, the outer-sphere reorganization energy, cannot in general be calculated explicitly for the structure of the individual molecule. We have used a conservatively low value of $0.3 \mathrm{eV}$ for the outer-sphere reorganization and computed the reorganization energy in the gas phase for each molecule (eq 2). As reorganization energies should not be strongly dependent on the morphology, ${ }^{52}$ we have used this simplified, outer reorganization energy across all crystal morphologies. The value of our calculated mobilities is uncertain to within approximately the same factor for all systems as the outer-sphere reorganization energy is in essence a scaling parameter that would affect all systems to a similar degree and thus not change any relative trends. In the screening, we only consider the inner reorganization energy $\left(\lambda=\lambda_{\text {inner }}^{\text {elec }}\right)$ in our $J / \lambda$ values, so those ratios will be overestimates of the true values. We note that while we cannot expect the absolute values to be correct, we can compare relative trends in which reorganization values are used. While the assumption of weak coupling (hopping regime) may not be valid in every case, it is most likely to be valid for low $J$ and, hence, low $J / \lambda$, so the screening methods should still be good at eliminating unpromising structures. We will discuss with care in the results section those systems that may not be adequately described by the hopping regime, but we always consider high $J$ values to be a condition for high mobility.

2.2. Crystal Structure Prediction. For the four most promising compounds with high $J_{\text {elec }} / \lambda_{\text {inner }}^{\text {elec }}$ ratios and electron mobilities, full CSP searches were performed to study the low-energy polymorphs observed on the crystal-energy landscape. This allowed validation of our assumption that the translational motif used for screening will occur in low-energy polymorphs of the candidate molecules. CSP also allows us to verify our dimer-model results by more sophisticated computational determination of the charge-carrier mobilities for the lowest-energy solid forms. Computational investigation of the polymorphic landscape and the associated charge mobilities rapidly provides information for new helicene compounds that could only be attained experimentally by a lengthy synthesis of the material, followed by (re)crystallizations and single-crystal mobility measurements.

The polymorphic energy landscapes of the top four candidate molecules identified in the screening process were generated using CrystalPredictorII, ${ }^{16}$ assuming a single molecule in the asymmetric unit $\left(Z^{\prime}=1\right)$ and searching the most common chiral and achiral space groups (see Section S3.1 for details). The energies of the putative crystalline structures were subsequently reranked using distributed multipole analysis (DMACRYS $\left.{ }^{53}\right)$. For all unique crystal structures within $15 \mathrm{~kJ} \mathrm{~mol}^{-1}$ of the energy minimum, single-point energies were computed using dispersion-corrected DFT. Specifically, the SIESTA ${ }^{54}$ implementation of the $\mathrm{B} 86 \mathrm{bPBE}^{55,56}$ functional and the exchangehole dipole-moment (XDM) dispersion correction ${ }^{57}$ was used with the double-zeta plus polarization (DZP) basis set. ${ }^{58}$ Single-point energies from B86bPBE-XDM/DZP have been shown to rapidly provide improved crystal-energy landscapes compared to distributed multipole analysis,${ }^{20}$ while full projector augmented-wave calculations using XDM are highly accurate for the intermolecular interactions within helicene crystals. ${ }^{31,32}$ Costly periodic relaxations of the crystals with DFT-XDM were omitted, due to time and computational cost constraints. Any future in-depth computational study of any of the screened polymorphs identified as interesting should begin with a full relaxation of the crystal structures before calculation of charge mobility properties. A more detailed description of the CSP methodology can be found in Section S3.

For the 10 lowest-energy DFT-ranked crystal structures of each compound, the charge-carrier mobilities were computed using nonadiabatic semi-classical Marcus theory, as described in Section S3.4. In contrast to the low-field limit mobility calculations based on a single dimer performed in the screening section, we computed the charge 

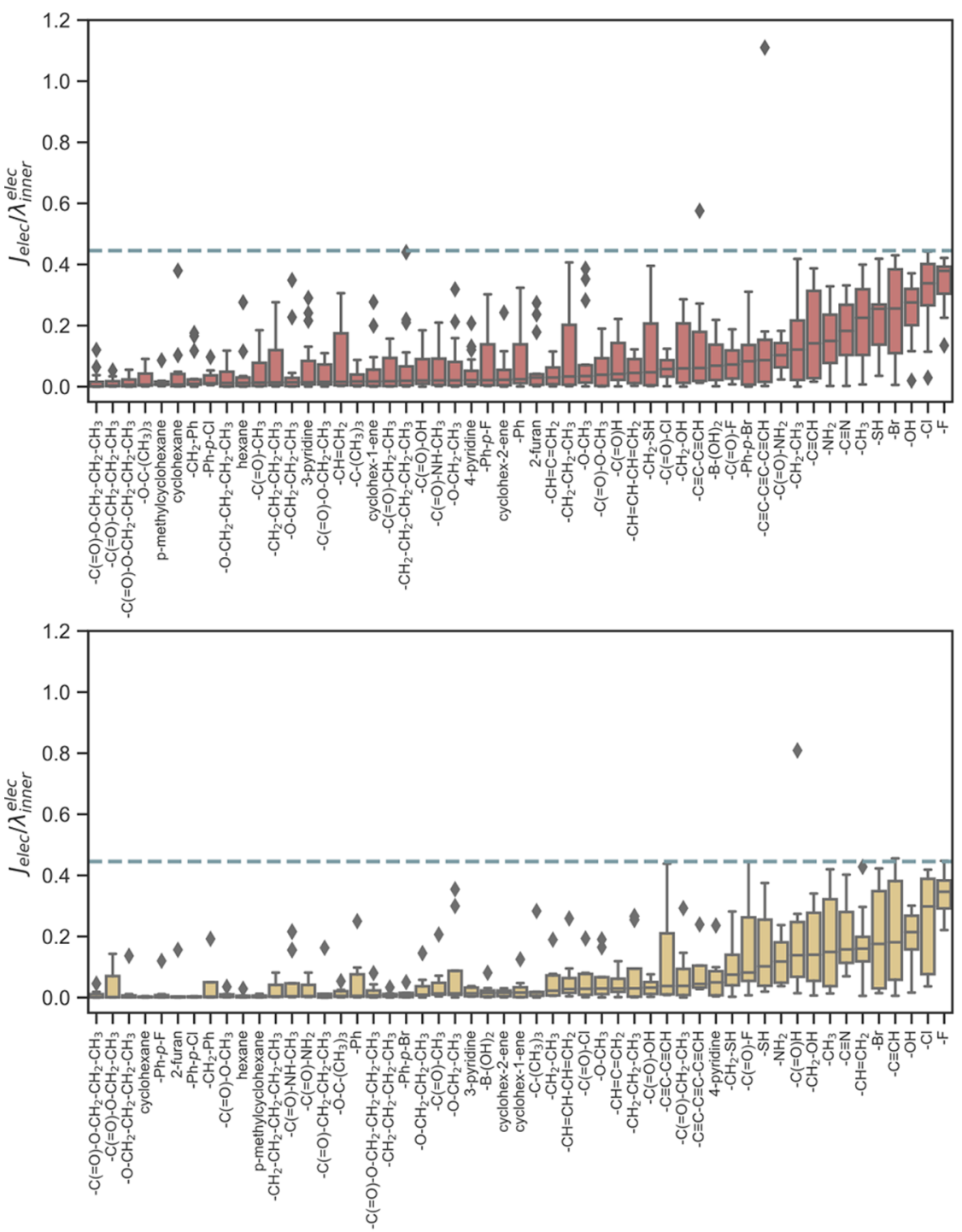

\section{Side Group}

Figure 4. Boxplots illustrating the OSC suitability of functionalized [6] helicene molecules, using $J_{\text {elec }} / \lambda_{\text {inner }}^{\text {elec }}$ as the target property. Results are shown for monosubstitution (top, red) or symmetric disubstitution (bottom, yellow) of the [6]helicene backbone. For each side group, 16 (monosubstituted) or 8 (disubstituted) data points were obtained, corresponding to all possible backbone sites. The size of the box is the interquartile range, containing $50 \%$ of the ratios, and the horizontal line inside each box is the median value. The whiskers indicate the range of ratios obtained outside the middle $50 \%$ of data points, with the exception of some large outliers, shown as gray diamonds. The dashed line is the unsubstituted [6] helicene reference. Figures S9 and S16 show the different components $(\lambda, J)$ of these data separately.

transport routes across the material in all three dimensions, for all dimers within a $15 \AA$ cutoff, thereby allowing a penalty for the outer reorganization energy, to yield a much more realistic representation of the charge-carrier mobility. Thus, in this second half of the discussion, we computed $\mu$ based on the CSP-generated crystals (Figure S32), and we included an outer reorganization energy of $\lambda_{\text {outer }}=0.3 \mathrm{eV}$, as explained above. Finally, when comparing crystal mobilities obtained for our new candidates with previous helicene results by Rice et al., ${ }^{32}$ we recomputed the mobilities using a $\lambda_{\text {outer }}=0.3 \mathrm{eV}$, to ensure that our mobilities can be qualitatively compared.

\section{RESULTS AND DISCUSSION}

3.1. OSC Suitability Screening. The transport properties of 1344 mono- and disubstituted helicene molecules were screened for potential OSC suitability using two different assessment criteria: (i) the straightforward $J_{\text {elec }} / \lambda_{\text {inner }}^{\text {elec }}$ ratio (hereafter referred to as $J / \lambda$ ) and (ii) the charge-carrier mobility, $\mu$, along the translational chain in the low-field limit.
Both approaches contain the transfer integral and inner reorganization energy; the mobility also explicitly considers the intermolecular center-of-mass distance of the dimer. In general, we aim to maximize the charge-transfer integral, $J_{\text {elec }}$ while minimizing the inner reorganization energy, $\lambda_{\text {inner }}^{\text {elec }}$ to yield optimal charge transport.

We note that we are assuming a hopping regime, which requires low $J / \lambda$ values to be valid. If anything, our approach, with $\lambda$ only accounting for the internal reorganization energy, is likely to overestimate $J / \lambda$ as an additional outer reorganization energy to the denominator would decrease the $J / \lambda$ values. Nevertheless, it appears likely that some of our systems with higher $J / \lambda$ values would no longer be within a hopping regime. If not in the hopping regime, we would expect the systems to be in a "transient localization" regime rather than in a band transport regime. However, in either case, the mobility should increase with the power of $J$ so that high $J$ 


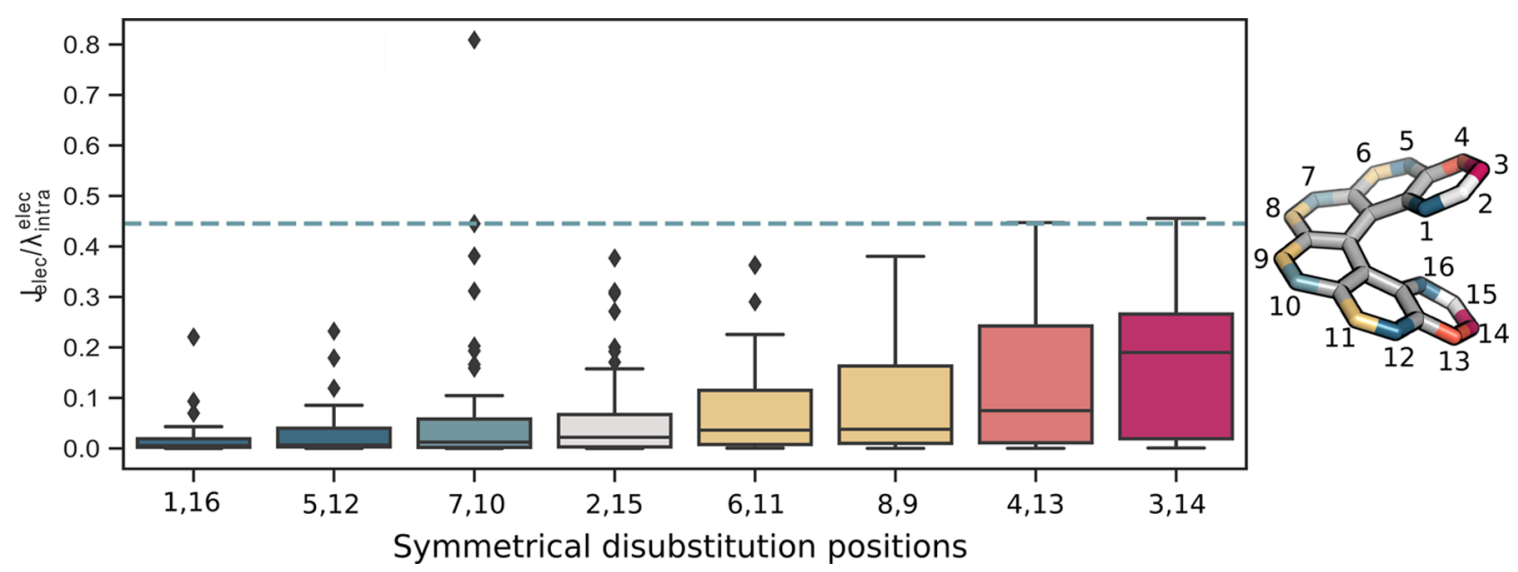

Figure 5. Boxplots illustrating ranges of the computed $J_{\text {elec }} / \lambda_{\text {inner }}^{\text {elec }}$ ratios spanning all 56 side groups. Results are shown for the eight symmetrical disubstitution patterns of the functionalized [6] helicene molecules, sorted in order of ascending median, and the coloring ranges from good charge transport (red) to no charge transport (blue). Significant outliers are shown as gray diamonds, and the dashed line is the unsubstituted [6]helicene reference. Analogous results for the monosubstituted [6] helicenes are shown in Figure S22.

values remain a necessary condition for good charge transport and, thus, remain a useful screening parameter. ${ }^{59}$ Figure S16 shows the distribution of $J$ values across the systems.

This screening provided insights into the impacts of side groups and substitution position(s) on electron transport. The calculated $J / \lambda$ ratios are shown in Figures 4 and 5 . The raw data is also available at https://data.hpc.imperial.ac.uk/ resolve/?doi=7858. Notably, there is no side group which consistently outperforms the carbo[6] helicene example. This is likely due to the steric hindrance of the translational dimer for some substitution positions. The $J / \lambda$ ratio generally increases toward the limiting value for the bare helicene (from left to right, Figure 4) because some substitution positions along the backbone are consistently disadvantageous for charge transport (independent of the side group attached), while in some positions, if the correct side group is attached, the terminally substituted [6] helicene has a larger intermolecular electronic coupling integral and thus a higher $J / \lambda$ ratio. Hence, the unfavorable substitution positions tend to be poor for any side group. However, for the better substitution positions, if a more favorable side group is attached, the $J / \lambda$ ratio increases in some, but not all, positions (thus, there is a larger spread of $J / \lambda$ values). Only a minority $(0.4 \%)$ of the $J / \lambda$ ratios computed for substituted [6] helicenes exceed the [6] helicene reference. This finding is of value in suggesting that while it may seem intuitively promising, the functionalization of [6] helicenes to improve electron transport is often not a fruitful approach in practice. This emphasizes the complexity of the multivariable problem of optimizing charge transport in organic semiconductors.

Overall, both screening metrics revealed three largely similar classes of terminal substituents, two of which give rise to electron transport: (i) small electronegative side groups (e.g., halogens and $\mathrm{CN}$ ), that give the best transport across all substitution patterns; (ii) moderately sized acyclic side groups with unsaturated bonds (diacetylene and triacetylene) that give rise to good transport for some substituent positions, usually manifesting as large outliers; and (iii) bulky alkyl substituents, cyclic or acyclic, that result in very poor transport. The same trends are observed for $\mu$ as for the simpler $J / \lambda$ ratio but are less pronounced. Across all substitution patterns and positions, we observe that smaller side groups, which do not heavily perturb the geometry of the [6] helicene backbone, perform better than bulky substituents (Figure 4). Bulky substituents are more likely to alter the inner strain and interplanar angle (the dihedral angle between the two terminal rings) of the [6] helicene backbone and thus affect electron delocalization across the backbone.

Despite their larger size, side groups containing double or triple bonds result in transfer integrals that are higher than expected from the general trend of an exponential decrease with increased molecular separation (Figure S15). In particular, triple bonds lead to favorably low inner reorganization energies, which shows that the ability to accommodate a negative charge is enhanced for these substituted [6] helicenes. This behavior is suggested by the LUMO being delocalized over the extended $\pi$-system introduced by the triacetylene unit (Figure S27). We find that this trend increases with the number of triple bonds per side group used for disubstitution (Figure S9 bottom). Functionalization strategies involving the inclusion of the triple-bonded 6,13-bis (triisopropylsilylethynyl) (TIPS) functional group to fine tune electronic properties have been used previously for other well-studied OSC molecular cores, such as pentacene $(\mathrm{Pn})^{60}$ or dinaphtho-fused $s$ indacenes. $^{61}$ TIPS side groups can increase a molecule's solubility, as well as directing the molecular packing, but without losing intermolecular electronic coupling at the same time. Molecular packing can be controlled by modifying the shape and size of the triple-bond-containing substituents. Depending on the ratio of the diameter of the side group to the pentacene backbone, different packing arrangements with altered intermolecular interaction strengths are observed. ${ }^{62,63}$ Triple-bond-containing side groups (e.g., TIPS-Pn, TMTES$\mathrm{Pn}$, diF-TESADT) can influence the distribution of intermolecular and intramolecular vibrational modes to minimize non-local electron-phonon coupling processes along the $\pi-\pi$ stacking direction (translational motif) that reduce the overall mobility. ${ }^{37}$ A longer discussion of the effect of $\pi$-system size on $\lambda_{\text {inner }}$ can be found in Section S2.6.1.

Fluorine substituents outperform any other side group for both mono- and disubstituted [6] helicenes when considering $J / \lambda$ ratios. This finding is consistent with previous research in leveraging electron-withdrawing halogens to lower the LUMO energy and the electron-injection barrier, such that electron and hole transport occurred simultaneously. ${ }^{64-66}$ Fluorination has been previously applied to enhance transport in other 
organic semiconductors, for example, arylene diimides, ${ }^{67,68}$ naphthalene, ${ }^{69}$ or functionalized acene derivatives, ${ }^{62}$ such as pentacene $^{70-72}$ or TIPS-pentacene. ${ }^{72}$ The impact of fluorination on reducing intermolecular spacing has been investigated for many OSC molecules, including carbon diimides, ${ }^{73}$ naphthalene, ${ }^{69}$ and benzene. ${ }^{74}$

Overall, we found that asymmetrical monosubstitution results in higher mean, median, and maximum transfer integrals than symmetrical disubstitution. Monosubstitution of [6] helicenes has been less experimentally investigated because the quickest and most common synthetic route to [6] helicenes is photocyclization, ${ }^{75}$ which gives symmetrically disubstituted products. The best side-group placements ranked by median were found to be the 3,14- and 4,13- positions for disubstitution and the 3-, 9-, and 13-positions for monosubstitution across all the side groups assessed (Figures 5 and S22).

The top-ranked helicenes for OSC performance, obtained from both metrics, are given in Table 1 . As stated above, the

Table 1. Top Three Candidates for the Mono- and Disubstituted [6] helicenes According to Both OSC Measures: $J_{\text {elec }} / \lambda_{\text {inner }}^{\text {elec }}$ Ratio and Low-Field Limit ChargeCarrier Mobility ${ }^{a}$

\begin{tabular}{ccccc}
$\begin{array}{c}\text { rank by } \\
J_{\text {elec }} / \lambda_{\text {inner }}^{\text {elec }}\end{array}$ & monosubstition & $J_{\text {elec }} / \lambda_{\text {inner }}^{\text {elec }}$ & disubstitution & $J_{\text {elec }} / \lambda_{\text {inner }}^{\text {elec }}$ \\
\hline $\mathbf{1}$ & 6-triacetylene & 1.11 & 7,10-dialdehyde & 0.81 \\
$\mathbf{2}$ & 7-diacetylene & 0.58 & 3,14-diethynyl & 0.46 \\
$\mathbf{3}$ & 2-chloro & 0.44 & 4,13-difluoro & 0.45 \\
$\mathbf{6}$ & 15-fluoro & 0.42 & & \\
$\mathbf{1 2}$ & 13-fluoro & 0.42 & & \\
rank by $\mu_{\text {elec }}$ & monosubstitution & $\mu_{\text {elec }}$ & disubstitution & $\mu_{\text {elec }}$ \\
\hline $\mathbf{1}$ & 6-triacetylene & 1.46 & 7,10-dialdehyde & 0.75 \\
$\mathbf{2}$ & 7-diacetylene & 0.38 & 3,14-diethynyl & 0.28 \\
$\mathbf{3}$ & 2-chloro & 0.26 & 4,13-difluoro & 0.27 \\
$\mathbf{8}$ & 15-fluoro & 0.24 & & \\
$\mathbf{1 4}$ & 13-fluoro & 0.24 & & \\
rank by $J_{\text {elec }}$ & monosubstitution & $J_{\text {elec }}$ & disubstitution & $J_{\text {elec }}$ \\
\hline $\mathbf{1}$ & 6-triacetylene & 251 & 7,10-dialdehyde & 191 \\
$\mathbf{2}$ & 7-diacetylene & 135 & 7,10-diacylfluoride & 118 \\
$\mathbf{3}$ & 15-fluoro & 74 & 5,11-diacylfluoride & 79 \\
$\mathbf{4}$ & 14-propylether & 73 & 4,13-difluoro & 78 \\
$\mathbf{2 9}$ & 13-fluoro & 71 & & \\
\hline & & & & \\
\hline
\end{tabular}

${ }^{a^{a}}$ The electron mobility $\mu_{\text {elec }}$ is given in $\times 10^{-3} \mathrm{~cm}^{2} \mathrm{~V}^{-1} \mathrm{~s}^{-1}$. The relevant $J_{\text {elec }}$ values are stated in $\mathrm{meV}$. fluorine substituent consistently gives the highest mean screening metrics, with 15-fluoro[6]helicene and 4,13difluoro[6]helicene being the best performers for mono- and di-substitution, respectively (13-fluoro[6] helicene is the second best monofluorination position). The other top-ranked cases consist of large outliers from the general trends, as shown in Table 1, and Figures 4 and 5. Across both screening metrics, the top three performing disubstituted helicenes were 7,10dialdehyde, 3,14-diethynyl, and 4,13-difluoro[6]helicene. Similarly, both measures gave 6-triacetylene, 7-diacetylene, and 2chloro[6]helicene as the best monosubstituted helicenes using the $J / \lambda$ ratio and the maximum low-field mobility. Giving consideration to the fact that the 6-triacetylene system with a high $J / \lambda$ value may no longer be in the hopping regime, we note that the system still has a high $J$ value (see Table 1 and Figure S16) that would be promising for charge transport in a "transient localization" regime. Thus, the selection of systems for further investigation would remain the same. In an ideal world, for systems where the hopping regime may no longer be valid, one would want to fully consider the factors that would be important in the "transient localization" regime. Namely, one would want information on the disorder in $J$ resulting from thermal fluctuations, the degree of anisotropy in these fluctuations, and the consequent effect on reducing mobility. ${ }^{76}$ It could, for example, be true that there are large thermal fluctuations in the 6-triacetylene system, causing anisotropic transport and thus reduced mobility. To gain a better insight into this, we would ideally want to calculate the intermolecular vibrational modes in each system, but this requires computationally demanding calculations where the full crystal structure is known, and is thus clearly beyond this screening study of over 1300 systems where the crystal structures are not a priori known.

Please refer to Supporting Information, Section S2.6, for an extended discussion of the screening results, covering how molecular structure affects electron transport (Section S2.6.1), the separation dependence of transfer integrals (Section S2.6.2), whether terminal substitution improves transfer integrals (Section S2.6.3), and whether triple bonds are good for transport (Section S2.6.4).

3.2. Analysis of the CSP Results. From the initial screening of OSC suitability, fluorine was found to be the best side group overall, independent of the substitution pattern and the exact functional group position. Thus, the highest performing disubstituted fluoro[6] helicenes, 4,13-difluoro[6]helicene, and the second highest and structurally related monofluorinated [6]helicene, 13-fluoro[6]helicene, were se-

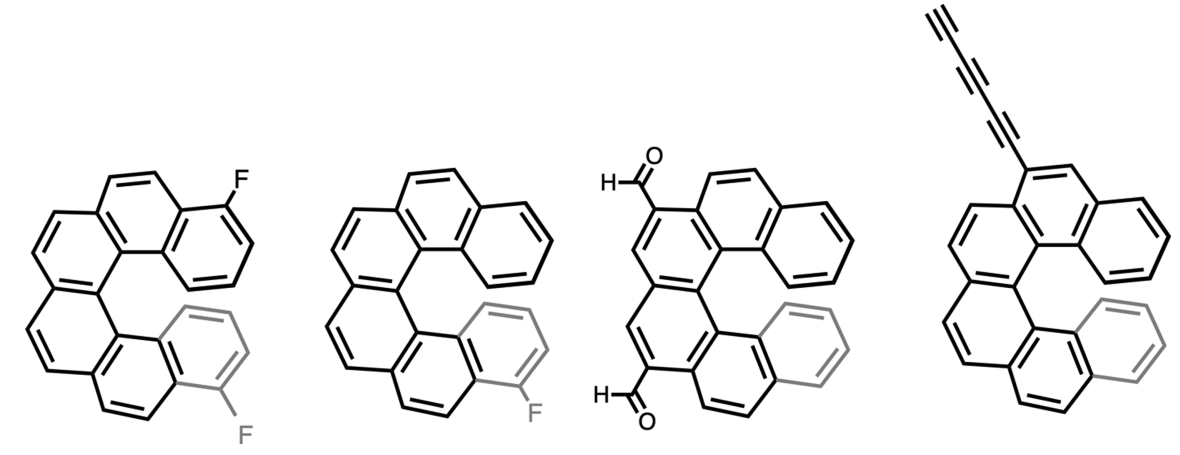

Figure 6. Four promising OSC molecular material candidates identified by computational screening. From left to right: 4,13-difluoro[6]helicene, 13-fluoro[6]helicene, 7,10-dialdehyde[6]helicene, and 6-triacetylene[6]helicene. 

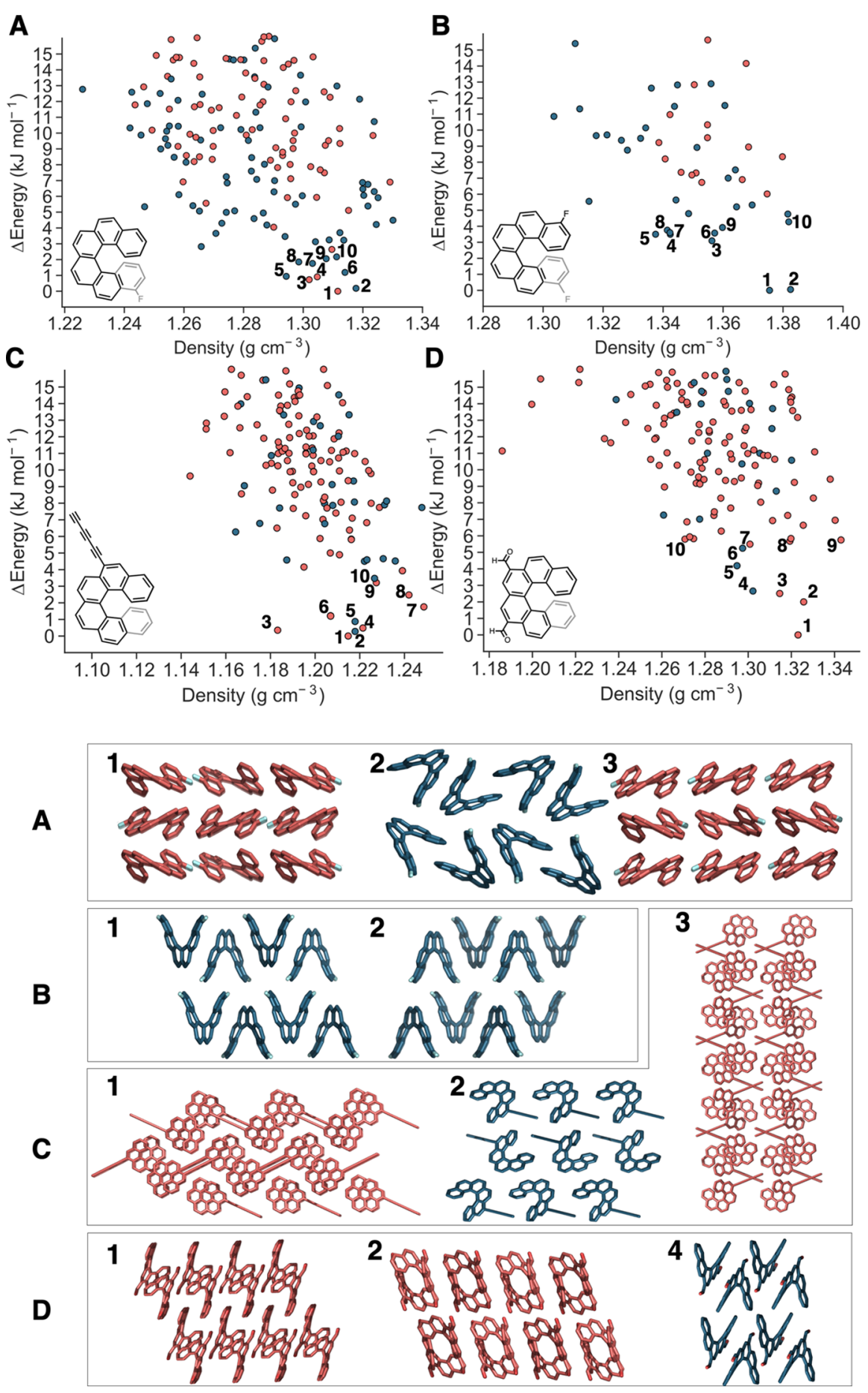

Figure 7. Top: Crystal-energy landscapes for 13-fluoro[6]helicene (A), 4,13-difluoro[6]helicene (B), 6-triacetylene[6]helicene (C), and 7,10dialdehyde[6] helicene (D). Crystals are plotted with their relative B86bPBE-XDM energy difference to the global minimum structure. This allows easy comparison of the thermodynamic stability of the solid forms. Data points are shown in blue (red) if they do (do not) contain the translational dimer motif. Low-energy crystals are numbered in order of ascending relative energy from the global minimum. The electron mobilities for the 10 lowest-energy polymorphs are reported in Figures 8, 9 and S32. Bottom: low-energy crystal packings, corresponding to the labeled structures above. Labeled crystal packings not shown here can be found in Figure S30. Oxygen atoms are shown in red and fluorine in light blue.

lected for CSP analysis. Additionally, the two extreme outlier cases in Table 1, 7,10-dialdehyde[6]helicene and 6triacetylene[6]helicene, were also selected for CSP. The structures of these four compounds are shown in Figure 6. For each of these candidate molecules, no solid-state structure has ever been synthesized, nor have mobilities been measured. However, our previous work has shown that CSP can provide predictions of experimentally realized helicene crystal structures, and we can qualitatively compare crystal mobilities using hopping theory, including explaining experimentally obtained properties. ${ }^{31,32}$ We note the caution with the application of the hopping regime to the 6-triacetylene case mentioned already above. To investigate further the capability of our approach to identify promising OSC molecular 


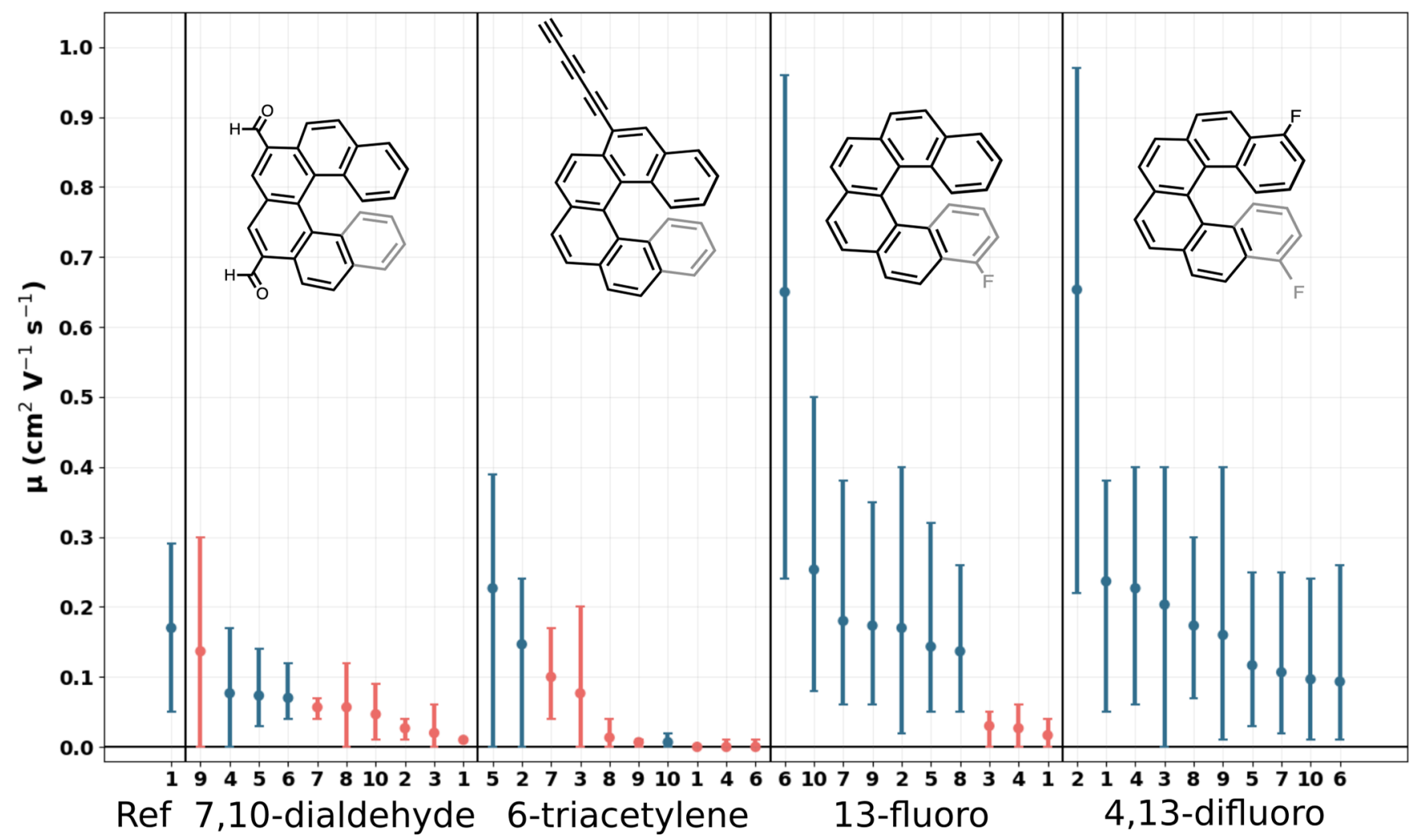

Figure 8. Minimum, maximum, and average electron mobilities, $\mu$, computed for each of the 10 lowest-energy polymorphs identified from the CSP searches. Data for structures with (without) the translational motif are shown in blue (red). Results for the reference [6] helicene crystal (HEXHEL) are shown on the far left, and for its 10 lowest-energy polymorphs in Figure S32.

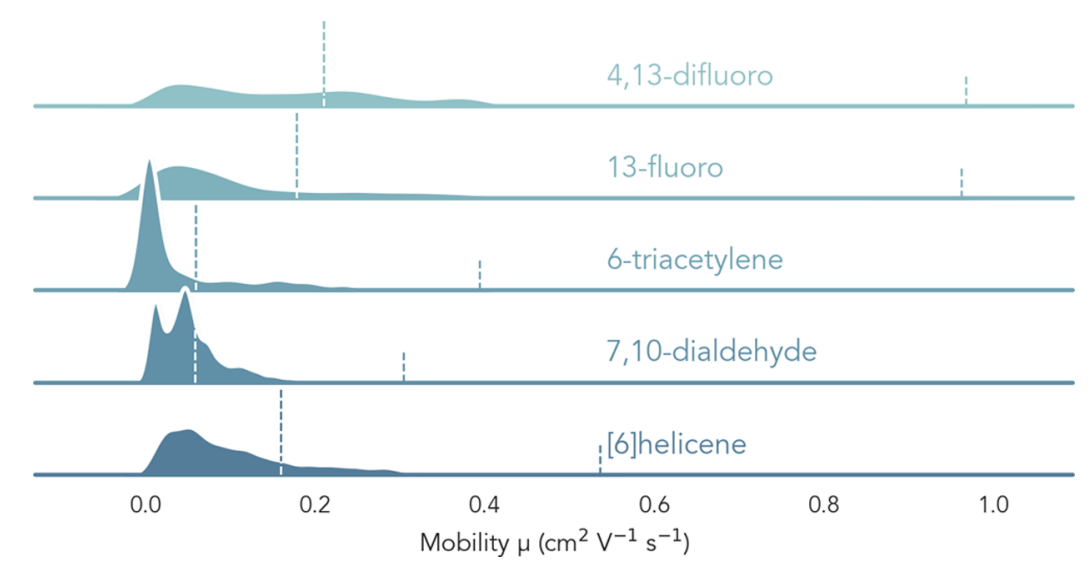

Figure 9. Distribution of the calculated electron mobilities observed across the 10 lowest-energy crystals for each molecule where CSP was performed ([6]helicene results as previously reported in ref 32). Maximum and average electron mobilities are indicated with dashed lines. Fluorination has the potential to improve charge transport in [6] helicenes, whereas 6-triacetylene and 7,10-dialdehyde[6]helicene perform worse than [6] helicene. The mobility plots for individual crystals can be found in Figures 8 and S32.

materials, we generated the low-energy crystal packings for the four selected candidates using CSP. The crystal-energy landscapes and resulting low-energy polymorphs are shown in Figure 7. In general terms, the lower in energy a polymorph is, the more likely it should be synthetically achievable.

As seen in Figure 7, the translational motif was repeatedly found in the crystal-energy landscape of the 13-fluoro[6]helicene and 4,13-difluoro[6] helicene molecules, with $68 \%$ and $46 \%$ frequency, respectively. For the cases with bulkier side groups, 7,10-dialdehyde and 6-triacetylene[6]helicenes, the translational motif was observed less frequently (18\% and $22 \%$, respectively). Among the 10 lowest-energy crystals in each
CSP landscape, the translational motif was observed $100 \%$ (4,13-difluoro), 70\% (13-fluoro), 30\% (6-triacetylene), and $30 \%$ (7,10-dialdehyde) of the time, compared to the [6] helicene reference (90\%). Fluorination does not affect the overall shape of the molecule and, thus, similar packing motifs (translational in one plane and back-to-back in the other plane) are observed as for [6]helicene. However, for the flexible dialdehyde and the large asymmetric triacetylene side group, the single-molecule geometry is significantly different from the [6] helicene backbone. Thus, it is not surprising that other motifs are accessed, with the translational motif seen less frequently. Additionally, due to the altered single-molecule 
geometry, the exact overlap angle of the translational motif and other motifs is changed slightly. It is, unfortunately, not the case that the translational dimer motif is guaranteed to be found, but it is plausible that a structure containing the motif can be found via a targeted experimental polymorph screening, and it does remain a frequently occurring one-dimensional packing motif in the helicene systems. From our previous CSP studies with helicenes, motifs frequently occurring in the calculated CSP landscapes are observed in experimentally obtained crystal packings ${ }^{32}$ and explain the experimentally observed properties of these systems. ${ }^{31}$

Figure 8 shows the electron mobilities across the 10 lowestenergy polymorphs of the global minimum for the CSP landscapes for the four molecules (i.e, those the most likely to be experimentally obtainable). Notably, in the case of fluorination, the translational motif gives rise to either the highest or second-highest transfer integral in the crystal polymorphs. Additionally, for both fluorinated [6]helicene CSP landscapes, the majority of the lowest-energy crystals contain the translational motif, while this motif is less prevalent in the higher-energy crystals. Across all four molecules, the translational motif coincides with comparably high mobilities. The distributions of the electron mobilities across the 10 lowest-energy polymorphs (in all planes of each polymorph) for each of the four CSP systems and for carbo[6]helicene are given in Figure 9. Both 6-triacetylene and 7,10-dialdehyde[6]helicene have a mean and maximum electron mobility significantly lower than the bare backbone. This suggests that neither of these modifications to the backbone are likely to enhance charge transport. In contrast, both fluorination examples have a higher mean and maximum electron mobility than for the bare backbone. Thus, it is the fluorinated systems where we are most hopeful of the synthesis of a polymorph with an increased charge-carrier mobility compared to the bare [6] helicene backbone.

4,13-Difluoro[6]helicene. For 4,13-difluoro[6] helicene, CSP found two polymorphs that are significantly lower in energy than the remaining candidate structures on the crystalenergy landscape (crystals $\mathbf{1}$ and 2, as shown in Figure 7B), and both contain the targeted translational motif. In fact, all 10 lowest-energy crystals contain the translational motif. The dimer model predicted a minimum-energy separation of $7.2 \AA$ for 4,13-difluoro[6] helicene, with a transfer integral of $J_{\text {elec }}=$ $78 \mathrm{meV}$. These properties are in excellent agreement with the CSP results, where the translational dimer gave rise to $J_{\text {elec }}=77$ $\mathrm{meV}$ at an intermolecular distance of $7.3 \AA$ for both low-energy polymorphs. This transfer integral is of the same order of magnitude as the [6] helicene reference dimer $(78 \mathrm{meV})$. For the lowest-energy crystal (1), the translational dimer gave rise to the highest transfer integral observed. Conversely, in crystal 2, the back-to-back dimer (Figure S1(VI,VII)) produced a higher transfer integral, of $133 \mathrm{meV}$, giving rise to a much higher overall electron mobility for this material (Figure S32). This compound is a particularly promising OSC candidate as both low-energy polymorphs give mobilities higher than the unsubstituted [6] helicene reference. Form 2 gives a significantly higher mobility than form $\mathbf{1}$ (the most stable structure). The two structures are nearly degenerate at the presented level of theory. Thus, form 2 could potentially become the most stable form if the crystal structures were DFT-D relaxed at a higher level of theory.

3.3. 13-Fluoro[6]helicene. Monofluorination gave rise to 176 low-energy polymorphs within $15 \mathrm{~kJ} \mathrm{~mol}^{-1}$ of the global minimum for 13-fluoro[6] helicene (Figure 7A), which is over three times as many as found for 4,13-difluoro[6] helicene. The asymmetry of 13-fluoro[6] helicene, relative to the symmetrically disubstituted molecule, stochastically increases the number of permutations of homo and heterochiral crystalline packings possible. The majority of 13-fluoro[6] helicene chiral crystals contained the translational motif (Figure S31(I)). 7 of the 10 lowest-energy polymorphs contained the translational motif at an average separation of $\bar{d}_{\min }=7.35 \AA$. For the two lowest-energy crystals containing the translational motif, 2 and 5, the intermolecular distances $(7.3 \AA)$ and maximum transfer integrals $(72 \mathrm{meV})$ for this motif are again in excellent agreement with the dimer screening prediction $\left(d_{\min }=7.3 \AA\right.$ and $J_{\text {elec }}=71 \mathrm{meV}$ ).

The mobilities of the 10 lowest-energy crystal structures for 13-fluoro[6]helicene reveal the influence of the translational motif on electron transport. The averaged maximum transfer integral of the polymorphs containing the translational motif (crystals $\mathbf{2}, \mathbf{5}-\mathbf{1 0}, \bar{J}_{\text {elec }}=80 \mathrm{meV}$ ) is significantly higher than the average obtained for the crystals without the translational motif (crystals 1, 3 and 4, $\bar{J}_{\text {elec }}=24 \mathrm{meV}$ ). Similarly, the averaged maximum electron mobilities are significantly improved if the translational motif is present, with $\bar{\mu}_{\max }$ computed to be 0.452 and $0.05 \mathrm{~cm}^{2} \mathrm{~V}^{-1} \mathrm{~s}^{-1}$ for crystals with and without the translational motif, respectively. The crystal with the highest mobility is polymorph 6 , which has $J_{\max }=128$ $\mathrm{meV}$ and $\mu_{\max }=0.96 \mathrm{~cm}^{2} \mathrm{~V}^{-1} \mathrm{~s}^{-1}$, leading to a $231 \%$ increase in the charge-carrier mobility compared to the [6] helicene reference structure ${ }^{33}\left(0.29 \mathrm{~cm}^{2} \mathrm{~V}^{-1} \mathrm{~s}^{-1}\right)$. While form 6 is not the thermodynamically most stable polymorphs considered in the mobility calculations, this polymorph is $<2 \mathrm{~kJ} \mathrm{~mol}^{-1}$ above the energetic minimum and may be isolable experimentally. Higher levels of theory, or inclusion of thermal free-energy corrections, could also substantially reorder the low-energy structures.

3.4. 6-Triacetylene[6]helicene. In this case, we can observe how our dimer screening model performs for helicenes with an altered overall molecular shape due to a large asymmetric perturbation to the backbone. In our initial screening, the 6-triacetylene[6]helicene translational dimer was found to have the shortest intermolecular distance (5.7 $\AA$ ) of all compounds considered. CSP confirms this abnormally short intermolecular distance, predicting values of $5.5 \AA$ for forms 2 and $\mathbf{5}$ (Table S3). However, due to the large side group, the translational dimer motif was present at a much lower frequency, occurring in $22 \%$ of the crystal structures. Where present, this motif did lead to promising charge mobility, with transfer integrals of $J_{\text {elec }}=125 \mathrm{meV}$ for form 2 and $J_{\text {elec }}=126 \mathrm{meV}$ for form 5, 76-77\% higher than the [6]helicene reference $(65 \mathrm{meV})$. This increase is potentially caused by the parallel $\pi-\pi$ stacking of the triacetylene side groups and helicene backbone at short intermolecular distances.

Crystal structures $\mathbf{5}$ and 2 , containing the translational dimer, produce the highest $\mu_{\max }$ and $\bar{\mu}$ across the 6-triacetylene polymorphs (Figure 9). The translational-type motif in crystal 6 gives rise to a lower mobility; however, as noted above, this crystal contains a heterochiral stacked dimer, with the side groups attached to opposite ends of the backbone, pointing in opposite directions. Despite the short intermolecular distances, only form 5 gives a maximum mobility $\left(0.39 \mathrm{~cm}^{2} \mathrm{~V}^{-1} \mathrm{~s}^{-1}\right)$ higher than the [6]helicene reference, and this is significantly lower than the results obtained for both the mono- and 
difluoro[6]helicenes. Consequently, crystal mobilities indicate poor electron transport for this screening candidate.

As discussed earlier, the high $J / \lambda$ values for this system are suggestive of the fact that it may not operate in the hopping regime, but more likely in the "transient localization" regime. Ideally, to understand the charge transport in this system, we would need to calculate the intermolecular vibrational modes to get an insight into thermal fluctuations that may reduce charge mobility. However, given there are already indications that this system is not promising at this point, the fact that these calculations are computationally demanding, and the CSP suggests multiple plausible low-energy polymorphs that would need to be considered, we do not believe this is a worthwhile effort, and certainly beyond the scope of this study.

3.5. 7,10-Dialdehyde[6]helicene. The CSP search for the flexible 7,10-dialdehyde[6] helicene molecule resulted in new packing motifs, most noticeably back-to-back dimers with the backbones overlapping to generate more of a network-type architecture (shown for form 1 in Figure 7D). Also, the 7,10dialdehyde[6]helicene is altered by the orientation of the carbonyl oxygens with respect to the initial gas phase dimers. Thus, the translational motif is slightly altered but still found in $18 \%$ of the crystal structures and $30 \%$ of the 10 lowest-energy structures. None of the 10 lowest-energy crystals lead to improved results compared to [6]helicene. The 7,10dialdehyde[6] helicene crystals perform even worse than the 6-triacetylene[6]helicene crystals, with lower average and maximum mobilities observed (Figures 9 and S32). While the vast majority of crystals do not show any measurable electron transport, the translational dimer is only observed across polymorphs that exhibit some weak transport, with, for example, crystals 5 and 4 have maximum mobilities of 0.14 and $0.17 \mathrm{~cm}^{2} \mathrm{~V}^{-1} \mathrm{~s}^{-1}$, respectively, compared to crystals 1,2 , and 3, which have very low mobilities in the range of $0.01-0.06 \mathrm{~cm}^{2}$ $\mathrm{V}^{-1} \mathrm{~s}^{-1}$.

\section{CONCLUSIONS}

In this work, 1344 monosubstituted and symmetrically disubstituted [6]helicenes were screened to assess their potential as OSC materials. The initial screening was based on properties of a specific homochiral translated molecular dimer, which is the most common motif found in low-energy polymorphs of carbo[6] helicene, and is also prevalent in other helicene structures present in the CSD. This dimer-based approach is orders of magnitude cheaper computationally than performing full CSP workflows for all candidates. However, due to the simplifications required in a screening approach for a complex multivariable problem such as this, it could misclassify systems that do not present with the assumed packing motif, or it may lead to incorrect ranking of systems that lie outside the assumed hopping regime of charge transport. Nonetheless, the fluorine side group, predicted by the screening to be the most promising substituent to maximize OSC performance metrics overall, was found to have promising electron mobilities when the predicted crystal polymorphs were analyzed.

In the 10 lowest-energy polymorphs of the CSP landscapes for the four most promising systems, the translational motif was found in $100 \%$ (4,13-difluoro), $70 \%$ (13-fluoro), 30\% (6triacetylene), and $30 \%$ (7,10-dialdehyde) of the structures. This is encouraging for the use of our dimer-based model, in that we could hope an experimental polymorph screening study could lead to the targeted motif. The intermolecular distances for the translational motif found in the lowest-energy crystal polymorphs were found to closely match the minimumenergy distances obtained from semi-empirical calculations on the isolated molecular dimers. Even the unusually shorter intermolecular distance $(5.7 \AA)$ in the 6-triacetylene[6]helicene dimer was confirmed through CSP. Although the translational-dimer motif did not necessarily yield the maximum transfer integral in the molecular crystals, its occurrence in low-energy polymorphs was strongly correlated to good electron transport properties. Using $J$ and $J / \lambda$ as metrics for charge-carrier mobility is a simplification and will be inadequate for strongly coupled systems. However, it serves as a first indicator of structures with promising transport properties. To fully characterize structures identified as promising from the screening approach, a phonon calculation of the relevant crystal structure would be an obvious next step. The dimer assumption is not perfect, and the frequency with which it was found for different systems does underline that there is still work to be done in finding a more general screening approach that is computationally viable for screening more than 1300 molecular systems, while also trying to factor in the complex interplay between molecular structure, solidstate structure, and charge-transport properties in the area of organic semiconductors. We do propose, however, that our approach is an improvement upon completely ignoring solidstate packing effects and performing computational screening only at the single-molecule level.

Comparing the present results to those for other [6] helicene OSCs, where we have used the same methodology to calculate electron transport indicators, ${ }^{31,32}$ one can clearly see that terminal substitution best improves the suitability of [6]helicenes for electron transport, while nitrogen substitution in aza[6] helicenes favors hole transport over electron transport. The maximum electron mobility calculated in aza[6] helicene crystals using our approach did not exceed $0.30 \mathrm{~cm}^{2} \mathrm{~V}^{-1} \mathrm{~s}^{-1}$ (4-aza[6] helicene: $0.26 \mathrm{~cm}^{2} \mathrm{~V}^{-1} \mathrm{~s}^{-1}$ ). ${ }^{33}$ Here, computational screening resulted in the identification of new candidate molecules with maximum electron mobilities more than three times greater than this limit using the same approach. Notably, some, but not all, 13-fluoro and 4,13-difluoro[6]helicene polymorphs outperform the unsubstituted carbo[6] helicene reference structure, with maximum mobilities of 0.96 and 0.97 $\mathrm{cm}^{2} \mathrm{~V}^{-1} \mathrm{~s}^{-1}$, respectively. Generally, of the four compounds carried forward from screening to CSP, the lowest-energy 4,13difluoro[6] helicene polymorphs most consistently exceeded the reference. The symmetric 4,13-difluoro[6]helicene is our most promising identified OSC compound, with both lowenergy polymorphs identified from the CSP possessing high electron mobilities; its synthesis should be feasible via a Wittig procedure starting from 2-fluorobenzaldehyde. Future work could include varying the degree of fluorination (tetra-, hexa-, octafluorination, etc.) to see whether this would further increase charge-carrier mobilities, similar to pentacenes. ${ }^{71}$

Overall, our screening approach to guide the design of new OSC molecular materials shows promise, even though it is short of a "universal solution" in organic semiconductor screening. Structural classification of the intermolecular packing into motifs was shown to be a powerful tool in the design of new functionalized materials, capable of providing observable crystal structure-mobility relationships. We hope that this approach can be developed further to aid in the extremely complex problem of accelerating the discovery of molecular materials with targeted properties. Our approach 
was able to assess only a minuscule fraction of the possibilities within a vast chemical space. Therefore, future screening approaches should also consider how to sample the search space more effectively and, in particular, how to generate "wild cards" that might not be suggested by humans. This may include generative models or inverse design approaches, although the complexity of the multivariable optimization required for OSCs, especially combined with the sensitivity of properties to molecular assembly, which again is extremely sensitive to molecular-level changes, makes this challenging. Any steps forward in the rapid prediction of molecular assembly, removing the need for simplified models such as our dimer approach here, would revolutionize the screening possible in this field.

\section{ASSOCIATED CONTENT}

\section{SI Supporting Information}

The Supporting Information is available free of charge at https://pubs.acs.org/doi/10.1021/acs.cgd.1c00473.

Computational methodologies, additional data derivation of the charge mobility in the low-field limit, and terminally substituted [6] helicenes CSD search results (PDF)

\section{AUTHOR INFORMATION}

\section{Corresponding Author}

Kim E. Jelfs - Department of Chemistry, Molecular Sciences Research Hub, Imperial College London, London W12 OBZ, U.K..; Centre for Processable Electronics, Imperial College London, London SW7 2AZ, U.K.; ๑ orcid.org/0000-00017683-7630; Email: k.jelfs@imperial.ac.uk

\section{Authors}

Julia A. Schmidt - Department of Chemistry, Molecular Sciences Research Hub, Imperial College London, London W12 OBZ, U.K..; 1 orcid.org/0000-0002-5934-3257

Joseph A. Weatherby - Department of Chemistry, Dalhousie University, Halifax, Nova Scotia B3H 4R2, Canada

Isaac J. Sugden - Department of Chemical Engineering, Imperial College London, London SW7 2AZ, U.K..

Alejandro Santana-Bonilla - Department of Chemistry, Molecular Sciences Research Hub, Imperial College London, London W12 OBZ, U.K..

Francesco Salerno - Department of Chemistry, Molecular Sciences Research Hub, Imperial College London, London W12 OBZ, U.K..; Centre for Processable Electronics, Imperial College London, London SW7 2AZ, U.K.

Matthew J. Fuchter - Department of Chemistry, Molecular Sciences Research Hub, Imperial College London, London W12 OBZ, U.K..; Centre for Processable Electronics, Imperial College London, London SW7 2AZ, U.K.; $\odot$ orcid.org/ 0000-0002-1767-7072

Erin R. Johnson - Department of Chemistry, Dalhousie University, Halifax, Nova Scotia B3H 4R2, Canada; (1) orcid.org/0000-0002-5651-468X

Jenny Nelson - Department of Physics and Centre for Processable Electronics, Imperial College London, London SW7 2AZ, U.K.

Complete contact information is available at: https://pubs.acs.org/10.1021/acs.cgd.1c00473

\section{Notes}

The authors declare no competing financial interest.

\section{ACKNOWLEDGMENTS}

We acknowledge funding from the European Research Council under FP7 (CoMMaD, ERC grant no. 758370), and the Engineering Research Council and Physical Sciences Research Council (EPSRC) (EP/P005543/1), including the UK's HEC Materials Chemistry Consortium (EP/L000202/1) for time on the UK supercomputer, ARCHER. K.E.J. thanks the Royal Society for a University Research Fellowship and for an Enhancement Award 2017 (J.A.S.). J.N. acknowledges funding from the European Research Council (CAPaCITy, Action no. 742708). M.J.F. acknowledges funding from the Centre for Processable Electronics (EP/L016702/1) and his EPSRC fellowship (EP/R00188X/1). The authors acknowledge funding from the Natural Sciences and Engineering Research Council (NSERC) of Canada to E.R.J. and from the Government of Nova Scotia for a fellowship to J.A.W. We also thank Compute Canada for computational resources. I.J.S. acknowledges the EPSRC (EP/J014958/1, EP/J003840/1, $\mathrm{EP} / \mathrm{P} 022561 / 1$, and EP/P020194) and the support of Eli Lilly. The authors thank Dr. Javier Segarra-Martí, Dr. Louise Price, and Prof. S. Claire Adjiman for useful discussions.

\section{REFERENCES}

(1) Bässler, H.; Köhler, A. Charge transport in organic semiconductors. Top. Curr. Chem. 2012, 312, 1-65.

(2) Petty, A. J.; Ai, Q.; Sorli, J. C.; Haneef, H. F.; Purdum, G. E.; Boehm, A.; Granger, D. B.; Gu, K.; Rubinger, C. P. L.; Parkin, S. R.; Graham, K. R.; Jurchescu, O. D.; Loo, Y.-L.; Risko, C.; Anthony, J. E. Computationally aided design of a high-performance organic semiconductor: The development of a universal crystal engineering core. Chem. Sci. 2019, 10, 10543-10549.

(3) Ruiz, C.; García-Frutos, E. M.; Hennrich, G.; Gómez-Lor, B. Organic semiconductors toward electronic devices: High mobility and easy processability. J. Phys. Chem. Lett. 2012, 3, 1428-1436.

(4) Kunkel, C.; Schober, C.; Margraf, J. T.; Reuter, K.; Oberhofer, H. Finding the Right Bricks for Molecular Legos: A Data Mining Approach to Organic Semiconductor Design. Chem. Mater. 2019, 31, 969-978.

(5) Nematiaram, T.; Padula, D.; Landi, A.; Troisi, A. On the Largest Possible Mobility of Molecular Semiconductors and How to Achieve It. Adv. Funct. Mater. 2020, 30, 2001906.

(6) Sorli, J. C.; Ai, Q.; Granger, D. B.; Gu, K.; Parkin, S.; Jarolimek, K.; Telesz, N.; Anthony, J. E.; Risko, C.; Loo, Y.-L. Impact of Atomistic Substitution on Thin-Film Structure and Charge Transport in a Germanyl-ethynyl Functionalized Pentacene. Chem. Mater. 2019, $31,6615-6623$.

(7) Musil, F.; De, S.; Yang, J.; Campbell, J. E.; Day, G. M.; Ceriotti, $\mathrm{M}$. Machine learning for the structure-energy-property landscapes of molecular crystals. Chem. Sci. 2018, 9, 1289-1300.

(8) Atahan-Evrenk, S.; Atalay, F. B. Prediction of Intramolecular Reorganization Energy Using Machine Learning. J. Phys. Chem. A 2019, 123, 7855-7863.

(9) Pulido, A.; Chen, L.; Kaczorowski, T.; Holden, D.; Little, M. A.; Chong, S. Y.; Slater, B. J.; McMahon, D. P.; Bonillo, B.; Stackhouse, C. J.; Stephenson, A.; Kane, C. M.; Clowes, R.; Hasell, T.; Cooper, A. I.; Day, G. M. Functional materials discovery using energy-structurefunction maps. Nature 2017, 543, 657-664.

(10) Campbell, J. E.; Yang, J.; Day, G. M. Predicted energystructure-function maps for the evaluation of small molecule organic semiconductors. J. Mater. Chem. C 2017, 5, 7574-7584.

(11) Motherwell, W. D. S.; Ammon, H. L.; Dunitz, J. D.; Dzyabchenko, A.; Erk, P.; Gavezzotti, A.; Hofmann, D. W. M.; Leusen, F. J. J.; Lommerse, J. P. M.; Mooij, W. T. M.; Price, S. L.; 
Scheraga, H.; Schweizer, B.; Schmidt, M. U.; Van Eijck, B. P.; Williams, D. E.; Williams, D. E. Crystal structure prediction of small organic molecules: A second blind test. Acta Crystallogr., Sect. B: Struct. Sci. 2002, 58, 647-661.

(12) Day, G. M.; Motherwell, W. D. S.; Ammon, H. L.; Boerrigter, S. X. M.; Della Valle, R. G.; Venuti, E.; Dzyabchenko, A.; Dunitz, J. D.; Schweizer, B.; Van Eijck, B. P.; Facelli, J. C.; Bazterra, V. E.; Ferraro, M. B.; Hofmann, D. W. M.; Leusen, F. J. J.; Liang, C.; Pantelides, C. C.; Karamertzanis, P. G.; Price, S. L.; Lewis, T. C.; Nowell, H.; Torrisi, A.; Scheraga, H. A.; Arnautova, Y. A.; Schmidt, M. U.; Verwer, P.; Verwer, P. A third blind test of crystal structure prediction. Acta Crystallogr., Sect. B: Struct. Sci. 2005, 61, 511-527.

(13) Day, G. M.; Cooper, T. G.; Cruz-Cabeza, A. J.; Hejczyk, K. E.; Ammon, H. L.; Boerrigter, S. X. M.; Tan, J. S.; Della Valle, R. G.; Venuti, E.; Jose, J.; Gadre, S. R.; Desiraju, G. R.; Thakur, T. S.; Van Eijck, B. P.; Bazterra, V. E.; Ferraro, M. B.; Hofmann, D. W. M.; Neumann, M. A.; Leusen, F. J. J.; Kendrick, J.; Price, S. L.; Misquitta, A. J.; Karamertzanis, P. G.; Welch, G. W. A.; Scheraga, H. A.; Arnautova, Y. A.; Schmidt, M. U.; van de Streek, J.; Wolf, A. K.; Schweizer, B.; Schweizer, B. Significant progress in predicting the crystal structures of small organic molecules - A report on the fourth blind test. Acta Crystallogr., Sect. B: Struct. Sci. 2009, 65, 107-125.

(14) Bardwell, D. A.; Adjiman, C. S.; Arnautova, Y. A.; Bartashevich, E.; Boerrigter, S. X. M.; Braun, D. E.; Cruz-Cabeza, A. J.; Day, G. M.; Della Valle, R. G.; Desiraju, G. R.; Van Eijck, B. P.; Facelli, J. C.; Ferraro, M. B.; Grillo, D.; Habgood, M.; Hofmann, D. W. M.; Hofmann, F.; Jose, K. V. J.; Karamertzanis, P. G.; Kazantsev, A. V.; Kendrick, J.; Kuleshova, L. N.; Leusen, F. J. J.; Maleev, A. V.; Misquitta, A. J.; Mohamed, S.; Needs, R. J.; Neumann, M. A.; Nikylov, D.; Orendt, A. M.; Pal, R.; Pantelides, C. C.; Pickard, C. J.; Price, L. S.; Price, S. L.; Scheraga, H. A.; Van De Streek, J.; Thakur, T. S.; Tiwari, S.; Venuti, E.; Zhitkov, I. K. Towards crystal structure prediction of complex organic compounds - A report on the fifth blind test. Acta Crystallogr., Sect. B: Struct. Sci. 2011, 67, 535-551.

(15) Day, G. M.; Motherwell, W. D.; Jones, W. A strategy for predicting the crystal structures of flexible molecules: The polymorphism of phenobarbital. Phys. Chem. Chem. Phys. 2007, 9, 16931704.

(16) Karamertzanis $\dagger$, P. G.; Pantelides, C. C. Ab initio crystal structure prediction. II. Flexible molecules. Mol. Phys. 2007, 105, 273-291.

(17) Beyer, T.; Lewis, T.; Price, S. L. Which organic crystal structures are predictable by lattice energy minimisation? CrystEngComm 2001, 3, 178-212.

(18) Hoja, J.; Ko, H.-Y.; Neumann, M. A.; Car, R.; DiStasio, R. A.; Tkatchenko, A. Reliable and practical computational description of molecular crystal polymorphs. Acta Hortic. Sin. 2019, 5, No. eaau3338.

(19) Brandenburg, J. G.; Grimme, S. Topics in Current Chemistry; Springer, 2014; Vol. 345, pp 1-24.

(20) Leblanc, L. M.; Johnson, E. R. Crystal-energy landscapes of active pharmaceutical ingredients using composite approaches. CrystEngComm 2019, 21, 5995-6009.

(21) Nyman, J.; Day, G. M. Static and Lattice Vibrational Energy Differences Between Polymorphs. CrystEngComm 2015, 17, 51545165.

(22) Whittleton, S. R.; Otero-de-la-Roza, A.; Johnson, E. R. Exchange-Hole Dipole Dispersion Model for Accurate Energy Ranking in Molecular Crystal Structure Prediction II: Non-Planar Molecules. J. Chem. Theory Comput. 2017, 13, 5332-5342.

(23) Sosorev, A. Y.; Maslennikov, D. R.; Kharlanov, O. G.; Chernyshov, I. Y.; Bruevich, V. V.; Paraschuk, D. Y. Impact of LowFrequency Vibrations on Charge Transport in High-Mobility Organic Semiconductors. Phys. Status Solidi RRL 2019, 13, 1970016.

(24) Gryn'Ova, G.; Lin, K. H.; Corminboeuf, C. Read between the Molecules: Computational Insights into Organic Semiconductors. J. Am. Chem. Soc. 2018, 140, 16370-16386.

(25) Brandt, J. R.; Salerno, F.; Fuchter, M. J. The added value of small-molecule chirality in technological applications. Nat. Rev. Chem. 2017, 1, 45 .
(26) Carpenter, J. E.; Grünwald, M. Heterogeneous interactions promote crystallization and spontaneous resolution of chiral molecules. J. Am. Chem. Soc. 2020, 142, 10755-10768.

(27) Kiran, V.; Mathew, S. P.; Cohen, S. R.; Hernández Delgado, I.; Lacour, J.; Naaman, R. Helicenes-A new class of organic spin filter. Adv. Mater. 2016, 28, 1957-1962.

(28) Isla, H.; Crassous, J. Helicene-based chiroptical switches. C. $R$. Chim. 2016, 19, 39-49.

(29) Jhulki, S.; Mishra, A. K.; Chow, T. J.; Moorthy, J. N. Helicenes as All-in-One Organic Materials for Application in OLEDs: Synthesis and Diverse Applications of Carbo-and Aza [5] helical Diamines. Chem.-Eur. J. 2016, 22, 9375-9386.

(30) Yang, Y.; Da Costa, R. C.; Fuchter, M. J.; Campbell, A. J. Circularly polarized light detection by a chiral organic semiconductor transistor. Nat. Photonics 2013, 7, 634-638.

(31) Yang, Y.; Rice, B.; Shi, X.; Brandt, J. R.; Correa da Costa, R.; Hedley, G. J.; Smilgies, D.-M.; Frost, J. M.; Samuel, I. D. W.; Oterode-la-Roza, A.; Johnson, E. R.; Jelfs, K. E.; Nelson, J.; Campbell, A. J.; Fuchter, M. J. Emergent Properties of an Organic Semiconductor Driven by its Molecular Chirality. ACS Nano 2017, 11, 8329-8338.

(32) Rice, B.; LeBlanc, L. M.; Otero-De-La-Roza, A.; Fuchter, M. J.; Johnson, E. R.; Nelson, J.; Jelfs, K. E. Correction: A computational exploration of the crystal energy and charge-carrier mobility landscapes of the chiral [6] helicene molecule. Nanoscale 2018, 10, $1865-1876$

(33) Salerno, F.; Rice, B.; Schmidt, J. A.; Fuchter, M. J.; Nelson, J.; Jelfs, K. E. The influence of nitrogen position on charge carrier mobility in enantiopure aza[6] helicene crystals. Phys. Chem. Chem. Phys. 2019, 21, 5059-5067.

(34) Szczypiński, F. T.; Bennett, S.; Jelfs, K. E. Can we predict materials that can be synthesised? Chem. Sci. 2020, 12, 830.

(35) Jansen, M.; Schön, J. C. Rational development of new materials-putting the cart before the horse? Nat. Mater. 2004, 3, 838.

(36) Gavezzotti, A.; Presti, L. L. Building Blocks of Crystal Engineering: A Large-Database Study of the Intermolecular Approach between $\mathrm{C}-\mathrm{H}$ Donor Groups and $\mathrm{O}, \mathrm{N}, \mathrm{Cl}$, or $\mathrm{F}$ Acceptors in Organic Crystals. Cryst. Growth Des. 2016, 16, 2952-2962.

(37) Illig, S.; Eggeman, A. S.; Troisi, A.; Jiang, L.; Warwick, C.; Nikolka, M.; Schweicher, G.; Yeates, S. G.; Henri Geerts, Y.; Anthony, J. E.; Sirringhaus, H. Reducing dynamic disorder in small-molecule organic semiconductors by suppressing large-amplitude thermal motions. Nat. Commun. 2016, 7, 10736.

(38) Roos, K.; Wu, C.; Damm, W.; Reboul, M.; Stevenson, J. M.; Lu, C.; Dahlgren, M. K.; Mondal, S.; Chen, W.; Wang, L.; Abel, R.; Friesner, R. A.; Harder, E. D. OPLS3e: Extending Force Field Coverage for Drug-Like Small Molecules. J. Chem. Theory Comput. 2019, 15, 1863-1874.

(39) Becke, A. D. A new mixing of Hartree-Fock and local densityfunctional theories. J. Chem. Phys. 1993, 98, 1372-1377.

(40) Frisch, M. J.; Trucks, G. W.; Schlegel, H. B.; Scuseria, G. E.; Robb, M. A.; Cheeseman, J. R.; Scalmani, G.; Barone, V.; Petersson, G. A.; Nakatsuji, H.; Li, X.; Caricato, M.; Marenich, A. V.; Bloino, J.; Janesko, B. G.; Gomperts, R.; Mennucci, B.; Hratchian, H. P.; Ortiz, J. V.; Izmaylov, A. F.; Sonnenberg, J. L.; Williams-Young, D.; Ding, F.; Lipparini, F.; Egidi, F.; Goings, J.; Peng, B.; Petrone, A.; Henderson, T.; Ranasinghe, D.; Zakrzewski, V. G.; Gao, J.; Rega, N.; Zheng, G.; Liang, W.; Hada, M.; Ehara, M.; Toyota, K.; Fukuda, R.; Hasegawa, J.; Ishida, M.; Nakajima, T.; Honda, Y.; Kitao, O.; Nakai, H.; Vreven, T.; Throssell, K.; Montgomery, J. A., Jr.; Peralta, J. E.; Ogliaro, F.; Bearpark, M. J.; Heyd, J. J.; Brothers, E. N.; Kudin, K. N.; Staroverov, V. N.; Keith, T. A.; Kobayashi, R.; Normand, J.; Raghavachari, K.; Rendell, A. P.; Burant, J. C.; Iyengar, S. S.; Tomasi, J.; Cossi, M.; Millam, J. M.; Klene, M.; Adamo, C.; Cammi, R.; Ochterski, J. W.; Martin, R. L.; Morokuma, K.; Farkas, O.; Foresman, J. B.; Fox, D. J. Gaussian 16, Revision C.01; Gaussian Inc., 2016.

(41) Landrum, G. RDKit Documentation, Read. Writ., 2011; Vol. 1, pp $1-79$.

(42) Bannwarth, C.; Ehlert, S.; Grimme, S. GFN2-xTB - An Accurate and Broadly Parametrized Self-Consistent Tight-Binding 
Quantum Chemical Method with Multipole Electrostatics and Density-Dependent Dispersion Contributions. J. Chem. Theory Comput. 2019, 15, 1652-1671.

(43) Neese, F. Software update: the ORCA program system, version 4.0. Wiley Interdiscip. Rev.: Comput. Mol. Sci. 2018, 8, No. e1327.

(44) Grimme, S.; Antony, J.; Ehrlich, S.; Krieg, H. A consistent and accurate $a b$ initio parametrization of density functional dispersion correction (DFT-D) for the 94 elements H-Pu. J. Chem. Phys. 2010, 132, 154104.

(45) Xie, X.; Santana-Bonilla, A.; Troisi, A. Nonlocal electronphonon coupling in prototypical molecular semiconductors from first principles. J. Chem. Theory Comput. 2018, 14, 3752-3762.

(46) Sakanoue, K.; Motoda, M.; Sugimoto, M.; Sakaki, S. A molecular orbital study on the hole transport property of organic amine compounds. J. Phys. Chem. A 1999, 103, 5551-5556.

(47) Chen, T.; Guestrin, C. XGBoost A scalable tree boosting system. Proc. ACM SIGKDD International Conference on Knowledge Discovery and Data Mining, 2016; pp 785-794.

(48) Robert, C. Machine Learning, a Probabilistic Perspective; MIT Press, 2014.

(49) Rogers, D.; Hahn, M. Extended-connectivity fingerprints. J. Chem. Inf. Model. 2010, 50, 742-754.

(50) Marcus, R. A. Chemical and electrochemical electron-transfer theory. Annu. Rev. Phys. Chem. 1964, 15, 155-196.

(51) Marcus, R. A. Electrostatic free energy and other properties of states having nonequilibrium polarization. I. J. Chem. Phys. 1956, 24, 979-989.

(52) Andrienko, D.; Kirkpatrick, J.; Marcon, V.; Nelson, J.; Kremer, K. Structure-charge mobility relation for hexabenzocoronene derivatives. Phys. Status Solidi 2008, 245, 830-834.

(53) Price, S. L.; Leslie, M.; Welch, G. W. A.; Habgood, M.; Price, L. S.; Karamertzanis, P. G.; Day, G. M. Modelling organic crystal structures using distributed multipole and polarizability-based model intermolecular potentials. Phys. Chem. Chem. Phys. 2010, 12, 84788490.

(54) Soler, J. M.; Artacho, E.; Gale, J. D.; García, A.; Junquera, J.; Ordejón, P.; Sánchez-Portal, D. The SIESTA method forab initioorder-Nmaterials simulation. J. Phys.: Condens. Matter 2002, 14, 2745.

(55) Becke, A. D. On the large-gradient behavior of the density functional exchange energy. J. Chem. Phys. 1986, 85, 7184-7187.

(56) Perdew, J. P.; Burke, K.; Ernzerhof, M. Generalized gradient approximation made simple. Phys. Rev. Lett. 1996, 77, 3865-3868.

(57) Johnson, E. R. In Non-covalent Interact. Quantum Chem. Phys; Otero-de-la Roza, A., DiLabio, G. A., Eds.; Elsevier, 2017, Chapter 5, pp 169-194.

(58) Leblanc, L. M.; Weatherby, J. A.; Otero-De-La-Roza, A.; Johnson, E. R. Non-Covalent Interactions in Molecular Crystals: Exploring the Accuracy of the Exchange-Hole Dipole Moment Model with Local Orbitals. J. Chem. Theory Comput. 2018, 14, 5715-5724.

(59) Fratini, S.; Mayou, D.; Ciuchi, S. The transient localization scenario for charge transport in crystalline organic materials. Adv. Funct. Mater. 2016, 26, 2292-2315.

(60) Griffith, O. L.; Anthony, J. E.; Jones, A. G.; Lichtenberger, D. L. Electronic properties of pentacene versus triisopropylsilylethynylsubstituted pentacene: Environment-dependent effects of the silyl substituent. J. Am. Chem. Soc. 2010, 132, 580-586.

(61) Zeidell, A. M.; Jennings, L.; Frederickson, C. K.; Ai, Q.; Dressler, J. J.; Zakharov, L. N.; Risko, C.; Haley, M. M.; Jurchescu, O. D. Organic semiconductors derived from dinaphtho-fused sindacenes: How molecular structure and film morphology influence thin-film transistor performance. Chem. Mater. 2019, 31, 6962-6970.

(62) Dong, H.; Wang, C.; Hu, W. High performance organic semiconductors for field-effect transistors. Chem. Commun. 2010, 46, 5211-5222.

(63) Wang, C.; Liu, Y.; Ji, Z.; Wang, E.; Li, R.; Jiang, H.; Tang, Q.; $\mathrm{Li}, \mathrm{H}$.; Hu, W. Cruciforms: assembling single crystal micro-and nanostructures from one to three dimensions and their applications in organic field-effect transistors. Chem. Mater. 2009, 21, 2840-2845.
(64) Zhang, B.; Kan, Y.-H.; Geng, Y.; Duan, Y.-A.; Li, H.-B.; Hua, J.; $\mathrm{Su}, \mathrm{Z} . \mathrm{M}$. An efficient strategy for improving carrier transport performance - Introducing fluorine into aryl substituted tetracene. Org. Electron. 2013, 14, 1359-1369.

(65) Tang, M. L.; Oh, J. H.; Reichardt, A. D.; Bao, Z. Chlorination: a general route toward electron transport in organic semiconductors. J. Am. Chem. Soc. 2009, 131, 3733-3740.

(66) Li, M.-J.; Long, M.-Q.; Chen, K.-Q.; Xu, H. Fluorination effects on the electronic transport properties of dithiophene-tetrathiafulvalene (DT-TTF) molecular junctions. Solid State Commun. 2013, 157, $62-67$.

(67) Jones, B. A.; Facchetti, A.; Wasielewski, M. R.; Marks, T. J. Tuning orbital energetics in arylene diimide semiconductors. Materials design for ambient stability of n-type charge transport. J. Am. Chem. Soc. 2007, 129, 15259-15278.

(68) Di Donato, E.; Fornari, R. P.; Di Motta, S.; Li, Y.; Wang, Z.; Negri, F. n-Type charge transport and mobility of fluorinated perylene bisimide semiconductors. J. Phys. Chem. B 2010, 114, 5327-5334.

(69) Maiti, B.; Schubert, A.; Sarkar, S.; Bhandari, S.; Wang, K.; Li, Z.; Geva, E.; Twieg, R. J.; Dunietz, B. D. Enhancing charge mobilities in organic semiconductors by selective fluorination: a design approach based on a quantum mechanical perspective. Chem. Sci. 2017, 8, 6947-6953.

(70) Koch, N.; Vollmer, A.; Duhm, S.; Sakamoto, Y.; Suzuki, T. The effect of fluorination on pentacene/gold interface energetics and charge reorganization energy. Adv. Mater. 2007, 19, 112-116.

(71) Kleemann, H.; Schuenemann, C.; Zakhidov, A. A.; Riede, M.; Lüssem, B.; Leo, K. Structural phase transition in pentacene caused by molecular doping and its effect on charge carrier mobility. Org. Electron. 2012, 13, 58-65.

(72) Tang, M. L.; Reichardt, A. D.; Wei, P.; Bao, Z. Correlating carrier type with frontier molecular orbital energy levels in organic thin film transistors of functionalized acene derivatives. J. Am. Chem. Soc. 2009, 131, 5264-5273.

(73) Purdum, G. E.; Telesz, N. G.; Jarolimek, K.; Ryno, S. M.; Gessner, T.; Davy, N. C.; Petty, A. J.; Zhen, Y.; Shu, Y.; Facchetti, A.; Collis, G. E.; Hu, W.; Wu, C.; Anthony, J. E.; Weitz, R. T.; Risko, C.; Loo, Y.-L. Presence of Short Intermolecular Contacts Screens for Kinetic Stability in Packing Polymorphs. J. Am. Chem. Soc. 2018, 140, $7519-7525$.

(74) Mottishaw, J. D.; Sun, H. Effects of Aromatic Trifluoromethylation, Fluorination, and Methylation on Intermolecular $\pi-\pi$ Interactions. J. Phys. Chem. A 2013, 117, 7970-7979.

(75) Shen, Y.; Chen, C.-F. Helicenes: synthesis and applications. Chem. Rev. 2012, 112, 1463-1535.

(76) Nematiaram, T.; Troisi, A. Strategies to reduce the dynamic disorder in molecular semiconductors. Mater. Horiz. 2020, 7, 29222928. 